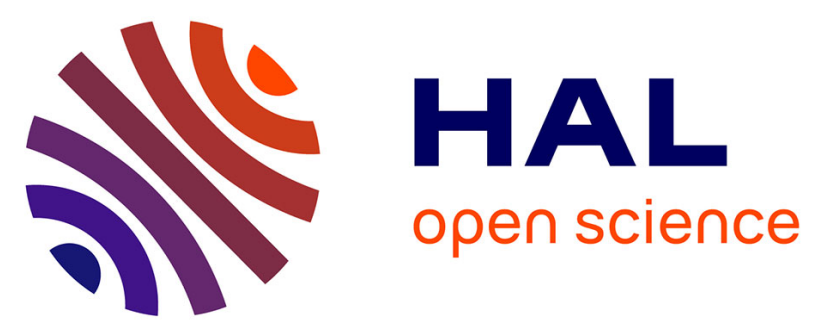

\title{
Modeling the effects of space structure and combination therapies on phenotypic heterogeneity and drug resistance in solid tumors
}

Alexander Lorz, Tommaso Lorenzi, Jean Clairambault, Alexandre Escargueil, Benoît Perthame

\section{To cite this version:}

Alexander Lorz, Tommaso Lorenzi, Jean Clairambault, Alexandre Escargueil, Benoît Perthame. Modeling the effects of space structure and combination therapies on phenotypic heterogeneity and drug resistance in solid tumors. Bulletin of Mathematical Biology, 2015, 77 (1), pp.1-22. 10.1007/s11538014-0046-4 . hal-00921266v2

\section{HAL Id: hal-00921266 \\ https://hal.sorbonne-universite.fr/hal-00921266v2}

Submitted on 12 Jan 2015

HAL is a multi-disciplinary open access archive for the deposit and dissemination of scientific research documents, whether they are published or not. The documents may come from teaching and research institutions in France or abroad, or from public or private research centers.
L'archive ouverte pluridisciplinaire HAL, est destinée au dépôt et à la diffusion de documents scientifiques de niveau recherche, publiés ou non, émanant des établissements d'enseignement et de recherche français ou étrangers, des laboratoires publics ou privés. 


\title{
Modeling the effects of space structure and combination therapies on phenotypic heterogeneity and drug resistance in solid tumors
}

\author{
Alexander Lorz ${ }^{123} \quad$ Tommaso Lorenzi ${ }^{123} \quad$ Jean Clairambault ${ }^{312}$ \\ Alexandre Escargueil ${ }^{45} \quad$ Benoît Perthame ${ }^{123}$
}

November 5, 2014

\begin{abstract}
Histopathological evidence supports the idea that the emergence of phenotypic heterogeneity and resistance to cytotoxic drugs can be considered as a process of selection in tumor cell populations. In this framework, can we explain intra-tumor heterogeneity in terms of cell adaptation to local conditions? Can we overcome the emergence of resistance and favor the eradication of cancer cells by using combination therapies? Bearing these questions in mind, we develop a model describing cell dynamics inside a tumor spheroid under the effects of cytotoxic and cytostatic drugs. Cancer cells are assumed to be structured as a population by two real variables standing for space position and the expression level of a phenotype of resistance to cytotoxic drugs. The model takes explicitly into account the dynamics of resources and anti-cancer drugs as well as their interactions with the cell population under treatment. We analyze the effects of space structure and combination therapies on phenotypic heterogeneity and chemotherapeutic resistance. Furthermore, we study the efficacy of combined therapy protocols based on constant infusion and/or bang-bang delivery of cytotoxic and cytostatic drugs.
\end{abstract}

\section{Introduction}

The levels of abiotic factors are not uniform throughout solid tumors [37, 39, 51, 52]. This leads to the creation of distinct niches differentiated by the local environment, which provide ecological opportunities for diversification (e.g., cells characterized by different proliferation or drug-resistant phenotypes can be selected depending on the local concentrations of nutrients and anti-cancer drugs), and paves the way for the emergence of intra-tumor heterogeneity [11, 16]. In this line, histopathological evidence supports the idea that the emergence of resistance to anti-cancer therapies can be considered as a process of Darwinian-like selection in tumor cell

\footnotetext{
${ }^{1}$ Sorbonne Universités, UPMC Univ Paris 06, UMR 7598, Laboratoire Jacques-Louis Lions, F-75005, Paris, France

${ }^{2}$ CNRS, UMR 7598, Laboratoire Jacques-Louis Lions, F-75005, Paris, France

${ }^{3}$ INRIA-Paris-Rocquencourt, EPC MAMBA, Domaine de Voluceau, BP105, 78153 Le Chesnay Cedex

${ }^{4}$ Sorbonne Universités, UPMC Univ Paris 06, F-75005, Paris, France

${ }^{5}$ INSERM, UMR_S 938, Laboratory of "Cancer Biology and Therapeutics", F-75012, Paris, France

Emails: alexander.lorz@upmc.fr, tommaso.lorenzi@upmc.fr, jean.clairambaulteinria.fr, alexandre.escargueileinserm.fr, benoit.perthame@upmc.fr
} 
populations $[16,32]$. In fact, malignant clones with heterogeneous genetic/epigenetic expressions leading to different phenotypes (e.g., epithelial vs mesenchymal, with the same genetic material [53]) can be seen as competing for space and resources (i.e., oxygen, glucose or other nutrients) within the environment defined by the surrounding tissues, together with the selective pressure exerted by therapeutic actions.

Cytotoxic drugs, such as DNA damaging agents and antimetabolites, are the most widely used chemical weapons in the fight against cancer. However, these drugs usually cause unwanted toxic side effects in the patients' organisms, since they are designed to kill cancer cells and are seldom specific toward tumor cells. Furthermore, they tend to kill strongly proliferative clones, usually considered as made of the most drug-sensitive cells [36], thus selecting for cells resistant to therapies $[17,18,24,46]$. These are the two major obstacles - toxic side effects and emergence of resistant clones - encountered in the clinic when using cytotoxic agents in treating tumors.

This situation calls for therapy optimization, that is, identification of drug doses and design of optimal delivery schedules in multi-drug combinations, allowing for an effective control of cancer growth. As regards multi-drug combinations, a trend in modern treatment of cancers leads to combining cytotoxic and cytostatic drugs. Cytostatic drugs slow down proliferation of cancer cells by blocking growth factor receptors, or downstream intracellular pathways involved in proliferation (e.g., tyrosine kinase inhibitors) and have lower toxicity for healthy cells. This mode of therapy combination allows to attain the twofold goal of reducing toxicity and holding in check the multiplication of resistant clones [42, 48, 49, 50, 54].

As far as drug delivery schedules are concerned, it has been suggested that, among others, infusion protocols based on bang-bang control (i.e., those protocols in which drug delivery is alternatively switched on and off over time) can allow an effective control of tumor size [28]. We will consider here the case where tumor cells are exposed to rectangle-wave infusions of cytotoxic and/or cytostatic drugs at constant concentrations.

Motivated by these considerations, and focusing on an in vitro cancer cell population as reference system (i.e., not taking into account unwanted toxicity to healthy cells, which is a theme we had explored in a previous paper [29]), we propose a space and phenotype structured population model for selection dynamics under the effects of cytotoxic and/or cytostatic drugs. The model we design includes birth and death processes of cancer cells. Furthermore, it also takes explicitly into account the dynamics of resources and anti-cancer drugs as well as their interactions with the cell population under treatment.

Cancer cells are assumed to be organized in a non-vascularized and radially symmetric spheroid. The assumption of radial symmetry is consistent with experimental evidence on tumor spheroids of small size [56]. As a simplification, we state our model in one space dimension [8, 22]. In spite of this apparently simplified assumption, we do believe our model relevant to study small small avascular tumors and micrometastases [9, 44], which are believed to be the target of adjuvant chemotherapies, but also to tackle, as an initial step, highly complex macrotumor models targeted by metastatic chemotherapy $[3,4]$.

Cells are structured as a population by two non-negative real variables $x \in[0 ; 1]$ and $r \in[0 ; 1]$ standing, respectively, for the normalized expression level of a cytotoxic resistant phenotype and for the normalized linear distance from the center of the spheroid. This implies that we do not consider a cell to be necessarily either totally sensitive or totally resistant to a given drug; we rather introduce a continuous structuring variable describing resistance between 0 (highly sensitive) and 1 (highly resistant).

It should be noted that, compared to the model proposed in [29], the present one is able to 
mimic the simultaneous selection of several traits (i.e., the rise of phenotypic polymorphism) within the cancer cell population, which provides the basis for intra-tumor heterogeneity. The additional spatial structure variable $r$, together with the diffusion along the $r$-axis of nutrients and therapeutic drugs, are the key ingredients of this model that enable the emergence of such a heterogeneous scenario, which is close to the ones observed in biological experimentations $[7,16,45]$. An alternative way to obtain the emergence of intra-tumor heterogeneity has been proposed in [27], by considering sufficiently large mutations in the models from [29].

At this stage, let us stress that both structure variables, $x$ and $r$, have a well defined biological meaning, so that they can be evaluated by means of laboratory experiments. In particular, a cell resistance level can be measured either by the expression level of ABC transporter genes, that are known to be associated with resistance to the drug $[40,46]$, or by the minimal dose of each drug under consideration needed to kill a given percentage of the cell population [57].

Let us furthermore mention that the derivation of models able to include both selection and spatial dynamics, as the one presented here, is a key step toward a better comprehension of those mechanisms that underlie the evolution of ecological systems in general. These models can pave the way for interesting mathematical questions; see for instance $[1,5,34,35]$ and references therein.

The paper is organized as follows. In Section 2, we describe the mathematical model and the related underlying assumptions. Section 3 establishes the setup for numerical simulations and provides some considerations about the choice of the parameter functions and their biological consistency. Further details about the values of parameters can be found in Appendix B. Section 4 is devoted to study cell environmental adaptation in the framework of our model (i.e., how tumor cells adapt to the surrounding environment defined by nutrients and anti-cancer drugs). In particular, the evolution of phenotypic heterogeneity and chemotherapeutic resistance are analyzed in the presence of cytotoxic and cytostatic drugs. A qualitative mathematical justification for the results presented here is proposed in Appendix A. With the aim of supporting the design of optimal therapeutic strategies, in Section 5 we test the efficacy of therapeutic protocols based on constant infusion and/or bang-bang delivery (i.e., infusion schedules relying on bangbang control) of cytotoxic drugs, cytostatic drugs or combinations of cytotoxic and cytostatic drugs. Conclusions are drawn in Section 6, which provides also some ideas about future research perspectives.

\section{A structured population model for a tumor cell spheroid ex- posed to anti-cancer drugs}

We present a mathematical model for the dynamics of an in vitro population of tumor cells exposed to cytotoxic and cytostatic drugs. The cell population is assumed to be organized in a non-vascularized and radially symmetric spheroid, and to be structured by two non-negative real variables $x \in[0,1]$ and $r \in[0,1]$. The former represents the normalized expression level of a cytotoxic resistant phenotype (i.e., roughly speaking, the level of resistance to cytotoxic agents), while the latter stands for the planar distance of cells from the center of the spheroid, whose radius is assumed to be normalized in order to have unitary length.

Cancer cells proliferate through the consumption of nutrients - oxygen and glucose, since in this setting we do not consider the glycolytic phenotype (i.e., we do not distinguish between these two nutrients) - and die due to interpopulation competition for space and resources. We 
sum up the effects of cytotoxic drugs directly on mortality (i.e., in this simple setting, not involving the cell division cycle, we do not consider drug effects on cell cycle phase transitions [23]), while we let cytostatic drugs slow down cellular proliferation. Nutrients and drugs are assumed to be in quasi-stationary equilibrium (i.e., their dynamics is assumed to be much faster than the selection dynamics involving cancer cells), and their concentrations vary over $r$ due to diffusion, decay and consumption by cancer cells.

The population density of cancer cells is modeled by the function $n(t, r, x) \geq 0$, so that local and global population densities at time $t \in[0, T]$ are computed, respectively, as

$$
\varrho(t, r)=\int_{0}^{1} n(t, r, x) d x, \quad \varrho_{T}(t)=\int_{0}^{1} \varrho(t, r) r^{2} d r
$$

while the local average level of resistance $X(t, r)$, the global average level of resistance $\chi(t)$ and the related variance $\sigma^{2}(t)$ can be evaluated as

$X(t, r)=\int_{0}^{1} x \frac{n(t, r, x)}{\varrho_{T}(t)} d x, \quad \chi(t)=\int_{0}^{1} X(t, r) r^{2} d r, \quad \sigma^{2}(t)=\int_{0}^{1} \int_{0}^{1} x^{2} \frac{n(t, r, x)}{\varrho_{T}(t)} r^{2} d r d x-\chi(t)^{2}$.

In the present mathematical framework, the function $\sigma^{2}(t)$ provides a possible measure for intratumor phenotypic heterogeneity at time $t$. The function $s(t, r) \geq 0$ identifies the concentration of nutrients available to cells. The densities of cytotoxic and cytostatic drugs are described, respectively, by $c_{1}(t, r) \geq 0$ and $c_{2}(t, r) \geq 0$.

The dynamics of functions $n, s, c_{1}$ and $c_{2}$ is ruled by the following set of equations

$$
\begin{gathered}
\partial_{t} n(t, r, x)=\left[\frac{p(x)}{1+\mu_{2} c_{2}(t, r)} s(t, r)-d \varrho(t, r)-\mu_{1}(x) c_{1}(t, r)\right] n(t, r, x), \\
-\alpha_{s} \Delta s(t, r)+\left[\gamma_{s}+\int_{0}^{1} p(x) n(t, r, x) d x\right] s(t, r)=0, \\
-\alpha_{c_{1}} \Delta c_{1}(t, r)+\left[\gamma_{c_{1}}+\int_{0}^{1} \mu_{1}(x) n(t, r, x) d x\right] c_{1}(t, r)=0, \\
-\alpha_{c_{2}} \Delta c_{2}(t, r)+\left[\gamma_{c_{2}}+\mu_{2} \int_{0}^{1} n(t, r, x) d x\right] c_{2}(t, r)=0,
\end{gathered}
$$

with zero Neumann conditions at $r=0$ coming from radial symmetry, and Dirichlet boundary conditions at $r=1$

$$
s(t, r=1)=s_{1}, \quad \partial_{r} s(t, r=0)=0, \quad c_{1,2}(t, r=1)=C_{1,2}(t), \quad \partial_{r} c_{1,2}(t, r=0)=0,
$$

In the above equations, $\Delta$ stands for the Laplacian in polar coordinates and:

- The function $p(x)$ models the proliferation rate of cells expressing the resistance level $x$ due to the consumption of resources. The factor

$$
\frac{1}{1+\mu_{2} c_{2}(t, r)}
$$

mimics the effects of cytostatic drugs. The parameter $\mu_{2}$ models the average uptake rate and effect on proliferation of these drugs. 
- The parameter $d$ is the average death rate of cells due to the competition for limited space.

- The function $\mu_{1}(x)$ denotes the death rate of cells due to the consumption of cytotoxic drugs.

- The parameters $\alpha_{s}, \alpha_{c_{1}}$ and $\alpha_{c_{2}}$ model, respectively, the diffusion constants of nutrients, cytotoxic drugs and cytostatic drugs.

- The parameters $\gamma_{s}, \gamma_{c_{1}}$ and $\gamma_{c_{2}}$ represent the decay rate of nutrients, cytotoxic drugs and cytostatic drugs, respectively.

The total delivered dose of anti-cancer drugs is computed as

$$
\int_{0}^{T}\left[C_{1}(t)+C_{2}(t)\right] d t
$$

The equation (2.1) can be recast in the equivalent form

$$
\partial_{t} n(t, r, x)=R\left(x, \varrho(t, r), c_{1,2}(t, r), s(t, r)\right) n(t, r, x),
$$

in order to highlight the role played by the net growth rate of cancer cells, which is described by

$$
R\left(x, \varrho(t, r), c_{1,2}(t, r), s(t, r)\right):=\frac{p(x)}{1+\mu_{2} c_{2}(t, r)} s(t, r)-d \varrho(t, r)-\mu_{1}(x) c_{1}(t, r) .
$$

The following considerations and hypothesis are assumed to hold:

- With the aim of translating into mathematical terms the idea that expressing cytotoxic resistant phenotype implies resource reallocation (i.e., redistribution of energetic resources from proliferation-oriented tasks toward development and maintenance of drug resistance mechanisms), we assume $p$ to be decreasing

$$
p(\cdot)>0, \quad p^{\prime}(\cdot)<0 .
$$

- The effects of resistance to cytotoxic therapies are modeled by assuming function $\mu_{1}$ to be decreasing

$$
\mu_{1}(\cdot)>0, \quad \mu_{1}^{\prime}(\cdot)<0 .
$$

Further considerations and details about the definitions of $p(x)$ and $\mu_{1}(x)$ are provided in the following section.

\section{Setup for numerical simulations and model parametrization}

Numerical simulations are performed in MATLAB making use of an implicit-explicit finite difference scheme combined with a shooting method with $200 \times 200$ points on the square $[0,1] \times[0,1]$. For all simulations, the interval $[0, T]$ is selected as time domain, with time step $d t=0.1$.

We choose the boundary and initial conditions to be

$$
\begin{gathered}
s(t, r=1)=s_{1}>0, \quad c_{1,2}(t, r=1)=C_{1,2}(t), \\
n(t=0, \cdot, x)=n^{0}(\cdot, x):=C^{0} \exp \left(-(x-0.5)^{2} / \varepsilon\right) .
\end{gathered}
$$

The parameter $s_{1}$ stands for the infusion rate of nutrients; the positive real functions $C_{1,2}(t)$ model the infusion rates of cytotoxic/cytostatic drugs and are defined, case by case, according 
to the situation under investigation in Section 4 and Section 5. Since we are dealing with a tumor spheroid of small size and micro-tumors are often derived from a single cell clone, we set $C^{0} \ll 1$ and $\varepsilon \ll 1$. In particular, the choice (3.2) mimics a biological scenario where most of the cells are characterized by the same intermediate level of resistance to therapies at the beginning of observations (i.e., the cell population is almost monomorphic).

We use linear functions to model the proliferation rate $p(x)$ and the death rate $\mu_{1}(x)$, which is consistent with the method of measuring selection via multiple regression, that can be adapted from ecology $[6,19]$. In more detail, we set

$$
p(x):=a_{1}+a_{2}(1-x), \quad \mu_{1}(x):=b_{1}+b_{2}(1-x) .
$$

The above definitions satisfy the generic properties set in (2.6)-(2.7). The parameters $a_{1,2}$ and $b_{1,2}$ are constrained by the following conditions:

$$
\begin{gathered}
b_{1} \propto a_{1}, \quad b_{2} \propto a_{2}, \\
\int_{0}^{1} a_{1}+a_{2}(1-x) d x=0.231\left[\mathrm{day}^{-1}\right] \quad \Rightarrow \quad a_{2}=0.4620-2 a_{1}\left[\mathrm{day}^{-1}\right], \\
\int_{0}^{1} b_{1}+b_{2}(1-x) d x=0.8\left[\text { day }^{-1}\right] \quad \Rightarrow \quad b_{2}=1.6-2 b_{1}\left[\text { day }^{-1}\right] .
\end{gathered}
$$

Relations (3.4) represent a possible way to model the relation between the rate of regression under chemotherapy and the rate of tumor growth established by the Norton-Simon hypothesis [43]. Conditions (3.5)-(3.6) require the values of the average proliferation rate and the average mortality rate due to the effect of cytotoxic drugs to be equal to some values coming from experimental evidence $[10,19]$. We set $a_{1}=0.1\left[\right.$ day $\left.^{-1}\right]$ and $b_{1}=0.1\left[\right.$ day $\left.^{-1}\right]$, so that the orders of magnitude of these parameters are consistent with the existing literature [?, ?, ?]. The values of the other parameters are selected with exploratory aim.

\section{Study of cell environmental adaptation and phenotypic het- erogeneity}

For the model described in the previous section, we now study how tumor cells adapt to the surrounding environment defined by nutrients and anti-cancer drugs. Subsection 4.1 deals with cell dynamics without drugs, while in Subsection 4.2 we analyze the effects of constant infusions of cytotoxic and cytostatic drugs. Considerations about the evolution of intra-tumor heterogeneity are drawn in Subsection 4.3. A qualitative mathematical justification for phenotypic selection is provided by Appendix A. Details about the values of parameters are provided in Appendix B. In particular, we select the distribution presented in Fig. 1 as initial datum in order to mimic a biological scenario where most of the cells are characterized by the same intermediate level of resistance to therapies at the beginning of observations (i.e., the cell population is almost monomorphic at time $t=0$ ). 


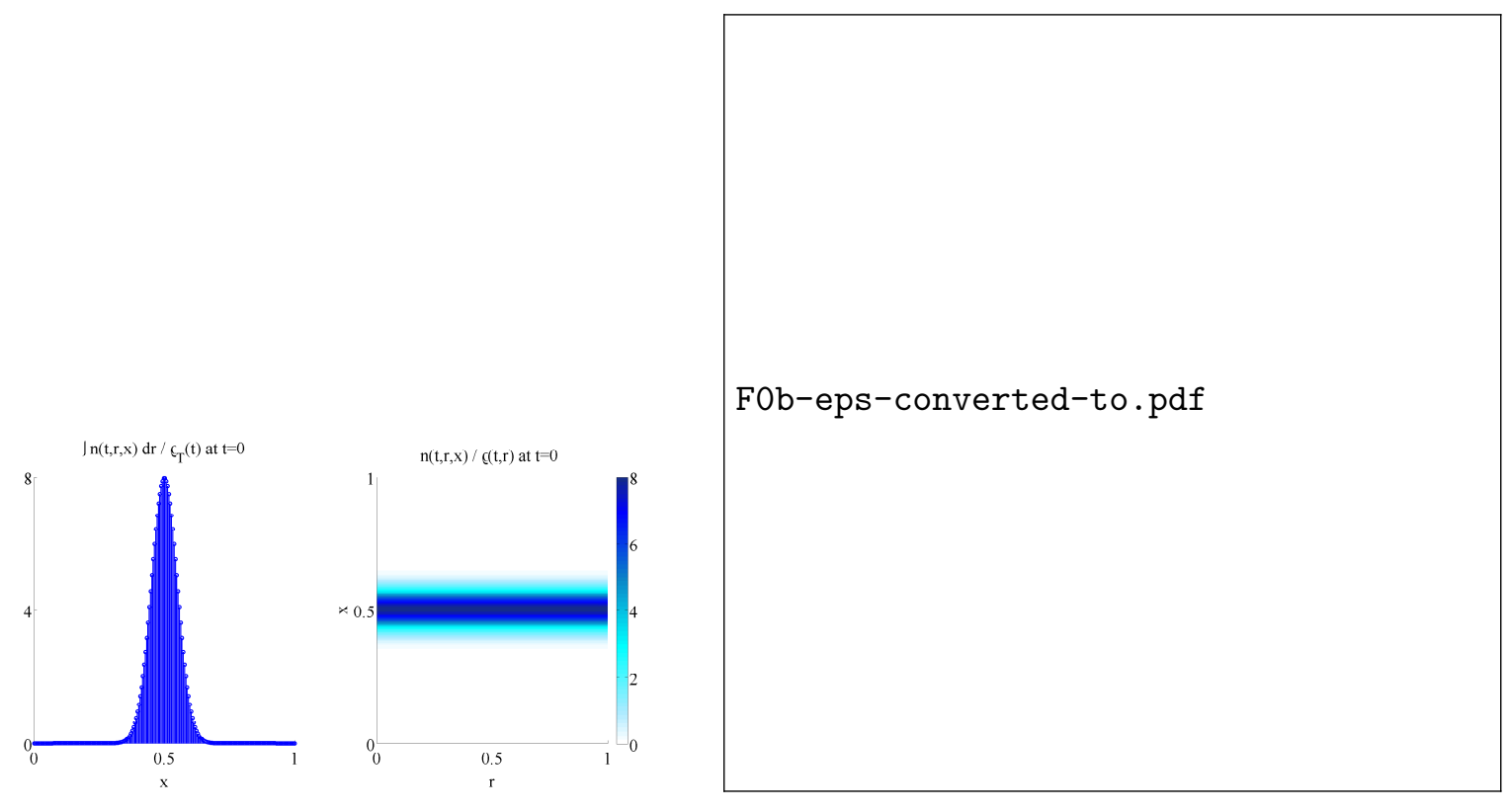

(a)

Figure 1: Initial cell distribution. Plots of $\int_{0}^{1} n(0, r, x) r^{2} d r / \varrho_{T}(0)$ (left panel) and $n(0, r, x) / \varrho(0, r)$ (right panel). The cell population is almost monomorphic at time $t=0$.

\subsection{Cell dynamics without therapies}

We begin our study by analyzing the dynamics of cancer cells without therapies $\left(i . e ., C_{1,2}(t):=\right.$ 0 ). The results obtained are summarized by Fig. 2(a), which shows how, in the absence of therapeutic agents, cells characterized by lower resistance levels and thus, due to assumption (2.6), by stronger proliferative potentials, are selected. At each position $r$, a different trait $X(T, r)$ is favored (i.e., for each value of $r, n(T, r, x)$ concentrates in a different point $X(T, r)$ ). In the framework of our model, this result can be justified by noting that the concentration of resources varies along the radius of the spheroid (i.e., $s(T, r)$ attains different values at each $r$, see solid lines in Fig. 3), and different densities of available nutrients imply the selection of different levels of ability to get resources, thus providing the basis for the emergence of polymorphism within the tumor cell population considered. This is in agreement with experimental evidence. In fact, it has been shown that growing spheroids display a proliferation gradient where only the external cells do proliferate [26]. These data are confirmed by in situ experiments where only tumor cells closed to vessels do proliferate in the absence of drugs [33].

\subsection{Cell dynamics under infusion of cytotoxic or cytostatic drugs}

At first, we consider the effects that constant infusions of cytotoxic drugs induce on cell dynamics, i.e., we perform simulations setting $C_{1}(t):=C_{1}>0$ and $C_{2}(t):=0$. Fig. 2(b) highlights how cytotoxic drugs promote a selective sweep toward resistant phenotypes. A polymorphic scenario arises at the end of simulations also in this case; in fact, a different level of resistance $X(T, r)$ is selected at any level within the spheroid (i.e., for each value of $r, n(T, r, x)$ concentrates in a 
different point $X(T, r))$. By analogy with the case without therapies, this is due to the fact that the concentrations of nutrients and cytotoxic drugs vary along the radius of the spheroid (i.e., $s(T, r)$ and $c_{1}(T, r)$ attain different values for different values of $r$, see solid and dashed lines in Fig. 3).

In order to study how cancer cells respond to the on-off switch of cytotoxic drug infusion, we perform simulations setting

$$
C_{1}(t):=\left\{\begin{array}{l}
C_{1}>0, \quad \text { if } t \in[0,20] \\
0, \quad \text { if } t \in(20,60],
\end{array}\right.
$$

and keeping $C_{2}(t):=0$. The above definition mimics a biological scenario where cytotoxic drugs are delivered in the time interval $[0, T / 3]$ only. Fig. 4 highlights the selection of higher levels of resistance during the infusion of cytotoxic drugs, i.e., on the time interval $[0, T / 3]$, and higher level of proliferative potential in the absence of cytotoxic drugs, i.e., on the time interval $(T / 3, T]$. In fact, when the infusion of cytotoxic drugs is stopped, more proliferative, and thus less resistant, cancer clones are favored [14, 15]. The switch from the selection for resistance to the selection for proliferative potential occurs in a progressive and continuous way, rather than through jumps in the distribution over the traits (see the right panel in Fig. 4).

Finally, we analyze the dynamics of cancer cells under the effects of constant infusions of cytostatic drugs, i.e., we run simulations setting $C_{1}(t):=0$ and $C_{2}(t):=C_{2}>0$ for any $t \in[0, T]$. Fig. 2(c) shows how the cell distribution at the end of simulations is still close to the initial one (see Fig. 1), that is, cytostatic drugs tend to slow down selection dynamics in the cancer cell population.

The results presented in this subsection lead us to conclude that phenotypic heterogeneity within solid tumors can come from cell adaptation to local conditions. In fact, cells characterized by different levels of proliferative potential and resistance to therapies can be selected depending on space position, in relation with the local concentrations of nutrients and anti-cancer drugs.

\subsection{Considerations about intra-tumor heterogeneity}

Here we study the evolution over time of the average level of resistance and the related variance. The results presented in Fig. 5 lead us to conclude that cytotoxic drugs cause an increase in time of the average level of resistance expressed by the whole cell population $\chi(t)$, while the related variance $\sigma^{2}(t)$ decreases. In the framework of our model, this result implies that cytotoxic drugs reduce intra-tumor heterogeneity. This is in agreement with the Gause competitive exclusion principle, and it is consistent with experimental observations for the fact that cytotoxic drugs reduce intra-tumor heterogeneity by favoring highly resistant cancer clones [12, 18, 25, 46].

In conclusion to this study of cell environmental adaptation, we observe that cytotoxic drugs tend to limit diversity by favoring only resistant clones. This results in a bottleneck effect, which decreases intra-tumor heterogeneity. 


\section{$5 \quad$ Study of optimized therapeutic protocols}

In this section, we compare the efficacy of different schedules of drug delivery with the aim of supporting the development of optimized therapeutic protocols. The effects of bang-bang infusion of cytotoxic or cytostatic drugs are compared with that of constant supply in Subsection 5.1, while the same kind of comparison for cytotoxic and cytostatic drugs in combination is provided in Subsection 5.2. Finally, the effects of therapeutic strategies that combine constant delivery of cytotoxic drugs with bang-bang infusion of cytostatic drugs, and vice-versa, are inspected in Subsection 5.3. The infusion schemes (i.e., the boundary conditions $C_{1,2}(t)$ ) in use throughout this section are summarized by Fig. 6, where the parameters $C_{a, b, c, d}$ model the instantaneous delivered doses. The values of these parameters are selected in such a way that the total delivered dose remains always the same.

\subsection{Constant vs bang-bang infusion of cytotoxic or cytostatic drugs only}

At first, we study the efficacy of therapeutic protocols relying on bang-bang delivery of cytotoxic drugs only, and we compare the results obtained with the ones of constant infusion. We perform simulations with $C_{2}(t):=0$ and $C_{1}(t)$ defined as in the center panel of Fig. 6 . The results obtained are compared with the outcomes of simulations performed with $C_{2}(t):=0$ and $C_{1}(t)$ defined as in the left panel of Fig. 6 .

Constant infusion of cytotoxic drugs leads to a temporary reduction of the population density of cancer cells, which is then followed by a relapse caused by the emergence of resistance, see Fig. 8(a). On the other hand, the bang-bang infusion scheme with the same total dose leads to a population evolution that is qualitatively the same as that observed in the absence of therapies, apart from a temporary reduction of the population density of cancer cells during drug delivery (see Fig. 8(b) and compare it with Fig. 7).

Then, we develop the same kind of analysis for cytostatic drugs only. We perform simulations with $C_{1}(t):=0$ and $C_{2}(t)$ defined as in the center panel of Fig. 6 . The results obtained are compared with the outcomes of simulations performed with $C_{1}(t):=0$ and $C_{2}(t)$ defined as in the left panel of Fig. 6. As we already know from Section 3, the constant infusion of cytostatic drugs tends to slow down the selection of high proliferative potentials by comparison with the case without drugs, see Fig. 9(a) and compare it with Fig. 7. On the other hand, the dynamics of cancer cells under bang-bang delivery of cytostatic drugs is qualitatively the same as that observed in the absence of therapies, apart from a temporary reduction of the population density of cancer cells during drug delivery (see Fig. 9(b) and compare it with Fig. 7).

As a conclusion to this section, we notice that the constant infusion of cytotoxic drugs leads to a temporary reduction of the population density of cancer cells, which is followed by a relapse caused by the emergence of resistance. As noted in the previous section, the constant infusion of cytostatic drugs slows down the dynamics of cancer cells. On the other hand, the bang-bang infusion of cytotoxic or cytostatic drugs weakly affects the dynamics of cancer cells by comparison

with the case without therapies, apart from temporary reductions of the population density of cancer cells during drug delivery. With the doses used in our tests, neither constant nor bang- 
bang infusion of only cytotoxic (cytostatic) drugs will push cancer cells toward extinction.

\subsection{Constant vs bang-bang infusion of cytotoxic and cytostatic drugs}

This subsection aims at making a comparison between the therapeutic effects of constant and bang-bang delivery of cytotoxic and cytostatic drugs in combination. Therefore, we perform simulations with $C_{1,2}(t)$ defined as in the center or the left panel of Fig. 6, respectively.

Bang-bang infusion leaves qualitatively unaltered the dynamics of cancer cells observed in the absence of therapeutic agents, apart from temporary reductions of the population density of cancer cells during drug delivery (see Fig. 10(b) and compare it with Fig. 7). On the other hand, the constant infusion scheme under consideration pushes cancer cells toward extinction, see Fig. 10(a). This is in agreement with the conclusions drawn in [29] and consistent with experimental observations suggesting that combination therapies can be more effective $[47,55]$. Therefore we are led to conclude that, keeping equal the total delivered dose of drugs, if cytotoxic and cytostatic agents are used in combination, protocols relying on simultaneous bang-bang infusion can be less effective than protocols relying on simultaneous constant infusion.

As a conclusion to this section, we observe that effective anti-cancer treatments can be designed by making use of proper combinations between cytotoxic and cytostatic drugs. If these drugs are delivered together, constant supply is more effective than bang-bang infusion and will push cancer cells toward extinction.

\subsection{Mixed constant/bang-bang infusions of cytotoxic and cytostatic drugs}

Finally, using the two types of drugs in combination, we test the effects of delivery schedules relying on constant infusion of cytotoxic drugs and bang-bang infusion of cytostatic drugs, or vice-versa. Therefore, we perform simulations with $C_{1,2}(t)$ as in the right panel of Fig. 6 . We define $C_{1}(t)$ as the fixed line and $C_{2}(t)$ as the dashed line, or vice-versa.

Bang-bang infusion of cytostatic drugs and constant infusion of cytotoxic drugs allows to control the tumor size, although a detectable number of cancer cells survives within the population, see Fig. 11(a). On the other hand, in good qualitative agreement with experimental observations [13], therapeutic protocols relying on bang-bang infusion of cytotoxic drugs and constant delivery of cytostatic drugs can push cancer cells toward extinction, see Fig. 11(b).

These results, together with that presented in Subsection 5.2, support the idea that more effective therapeutic protocols can be designed by using cytotoxic and cytostatic drugs in combination, with constant delivery for both drugs, or bang-bang infusion for cytotoxic drugs and constant infusion for cytostatic drugs. This can be justified by observing that cytostatic drugs in constant infusion slow down the emergence of resistance. As a consequence, the efficacy of cytotoxic drugs is enhanced, so that the same total dose of cytotoxic drugs can push cancer cells toward extinction.

As a conclusion to this section, we notice that therapeutic protocols relying on bang-bang infusion of cytotoxic drugs - constant delivery of cytostatic drugs are more effective than therapeutic protocols based on bang-bang infusion of cytostatic drugs - constant delivery of cytotoxic drugs. 
The former make possible to push cancer cells toward extinction, while the latter allow only a control on tumor size.

\section{Conclusions and perspectives}

Departing from theories derived in other contexts of population biology and Darwinian-like selection, we have developed a space and phenotype structured population model for the dynamics of cancer cells exposed to cytotoxic and cytostatic drugs. Relying on the assumption that cells are organized in a radially symmetric spheroid, the present model takes explicitly into account the dynamics of resources and anti-cancer drugs, which define the cellular environment. In the present model, space structure together with diffusion of nutrients and therapeutic agents are the key ingredients providing the basis for the emergence of intra-tumor heterogeneity (i.e., the simultaneous selection of several levels of resistance/proliferative potential within the cancer cell population).

\subsection{Study of cell environmental adaptation and phenotypic heterogeneity}

In the framework of this model, we have first made use of numerical simulations to analyze the evolution of phenotypic heterogeneity and the emergence of resistance to therapies (see Section $3)$, and we have reached the following conclusions:

- Phenotypic heterogeneity within solid tumors can be explained, at least partially, by cell adaptation to local conditions. In fact, cells characterized by different levels of proliferative potential and resistance to therapies are selected depending on space position, in relation with the distributions of resources and anti-cancer drugs.

- Cytostatic drugs can slow down tumor evolution, while cytotoxic drugs can favor the selection of highly resistant cancer clones and cause a decrease in the heterogeneity with respect to the resistance trait. In the framework of our model, this is not in contradiction with the Gause competitive exclusion principle.

\subsection{Study of optimized therapeutic protocols}

As a second step, we have tested, in silico, the capability of different therapeutic protocols to effectively contrast cancer progression. The cases of constant infusion, bang-bang delivery and mixed constant - bang-bang infusion of the anti-cancer drugs under study have been considered (see Section 4). The results we have obtained lead us to the following conclusions:

- Looking for combination therapies relying on cytotoxic and cytostatic drugs is a more effective strategy for fighting cancer rather than using high doses of cytotoxic or cytostatic drugs only.

- Therapeutic protocols relying on constant supply of cytotoxic and cytostatic drugs, or bangbang infusion of cytotoxic drugs and constant delivery of cytostatic drugs are more effective than the other protocols considered here, since they can push cancer cells toward extinction. This can be justified by noting that cytostatic drugs slow down the growth of resistant cells and, thus, cytotoxic drugs can kill more cancer cells in the same time window.

- Since constant supplies of cytotoxic drugs might be excluded in practice for toxicity reasons, 
the bang-bang delivery of cytotoxic drugs associated with the constant infusion of cytostatic drugs might turn out to be optimal.

\subsection{Perspectives}

Future researches will be addressed to extend the present model in order to include the dynamics of healthy cells, and study how to handle unwanted toxic side effects related to the delivery of anti-cancer agents. In view of this, a possible research direction is to approach the toxicity problem at stake as an optimal control problem, where the systemic cost for human body related to cancer growth and therapy infusion has to be minimized under the constraint that a minimal concentration of healthy cells should be preserved inside the system. In the same way, in view of adaptive therapy [15], preserving a minimal proportion of cancer cells under a sensitivity threshold $x \leq \bar{x}$ (to be tuned) is another possible constraint to be represented in an optimal control problem. This general direction of research, using optimal control settings, clearly aims at therapeutic optimization in the clinic of cancers.

From a modeling point of view, the present theoretical study has taken into account (additionally to the space variable $r$ ) only one drug resistance trait for two different classes of anticancer drugs. This study should also be completed in the future by further work involving a multidimensional structure variable $x$ including different resistant phenotypes to different classes of drugs. Furthermore, it might be interesting to consider other phenotypes related, for instance, to epithelial-to-mesenchymal transition, glycolytic metabolism and dormancy, which are involved in the emergence of cell subpopulations less accessible to treatment than the classical forms of in situ proliferating epithelial solid tumors.

From a more biological point of view, understanding what are the cell population characters (genetically or epigenetically determined?) aggregated in this structure variable $(r, x)$ standing for spatial heterogeneity and quantitated drug resistance, and how it can be related in experimental measurements with gene expression or epigenetic enzyme activity, is a big challenge that must be faced in transdisciplinary studies gathering mathematicians and biologists. We know from Luria and Delbrück's princeps experiment [31] that some drug resistance, due to stochastic genetic mutations in cell populations, occurring prior to drug exposure, is likely to

exist in cancer cell populations, all the more so as genome instability is a common feature of these cells $[2,21]$. Finding out what are the respective parts played in drug resistance by purely stochastic processes [20] on the one hand, and by more Lamarckian-like phenomena resulting from adaptation of the cells surviving a massive drug infusion, involving epigenetic mechanisms in response [41], and thus justifying the use of deterministic models, is another challenge that we intend to tackle in forthcoming studies.

Acknowledgments. The research leading to this paper was (partially) funded by the french "ANR blanche" project Kibord: ANR-13-BS01-0004. T.L. was supported by the Fondation Sciences Mathématiques de Paris and by a public grant overseen by the French National Research Agency (ANR) as part of the "Investissements d'Avenir" program (reference: ANR-10-LABX-0098), and by the FIRB project - RBID08PP3J 


\section{Appendix A. A qualitative mathematical justification for pheno- typic selection}

From a mathematical standpoint, using the considerations drawn in [30, 34, 38], the long term dynamics of $X(t, r)$ can be formally characterized by evaluating

$$
\lim _{t \rightarrow \infty} R\left(x=X(t, r), \varrho(t, r), c_{1,2}(t, r), s(t, r)\right)=0 .
$$

In order to verify it numerically, we write the following identity

$$
R\left(X(T, r), \varrho(T, r), c_{1,2}(T, r), s(T, r)\right)=0,
$$

which implies, due to the definitions of the functions $p$ and $\mu_{1}$ provided in Appendix B,

$X(T, r)=\frac{1+\mu_{2} c_{2}(T, r)}{a_{2} s(T, r)-b_{2} c_{1}(T, r)\left[1+\mu_{2} c_{2}(T, r)\right]}\left[\frac{s(T, r)}{1+\mu_{2} c_{2}(T, r)}\left(a_{1}+a_{2}\right)-d \varrho(T, r)-\left(b_{1}+b_{2}\right) c_{1}(T, r)\right]$.

Fig. 12 shows how the curves $X(T, r)$ obtained from the formula above are in good agreement with the positions of the maximum points $x_{M}(T, r)$ of $n(T, r, x) / \varrho(T, r)$ that result from numerical simulations.

\section{Appendix B. Values of the parameters}

Figure 2: $T=6000, \alpha_{s}=0.08, \alpha_{c_{1}}=0.08, \alpha_{c_{2}}=0.2, \gamma_{s}=1, \gamma_{c_{1}}=1, \gamma_{c_{2}}=1, \mu_{2}=800$, $d=0.3620$; (a) $s_{1}=0.1, C_{1}(t):=0, C_{2}(t):=0 ;$ (b) $s_{1}=0.3, C_{1}(t):=0.07, C_{2}(t):=0 ;(\mathrm{c})$ $s_{1}=0.3, C_{1}(t):=0, C_{2}(t):=0.07$.

Figure 3: $T=6000, \alpha_{s}=0.08, \alpha_{c_{1}}=0.08, \alpha_{c_{2}}=0.2, \gamma_{s}=1, \gamma_{c_{1}}=1, \gamma_{c_{2}}=1, s_{1}=0.3$, $\mu_{2}=800, d=0.3620 ;$ (left) $C_{1}:=0.07, C_{2}(t):=0 ;$ (right) $C_{1}:=0, C_{2}(t):=0.07$.

Figure 4: $T=3000, \alpha_{s}=0.08, \alpha_{c_{1}}=0.08, \alpha_{c_{2}}=0.2, \gamma_{s}=1, \gamma_{c_{1}}=1, \gamma_{c_{2}}=1, s_{1}=0.3$, $\mu_{2}=800, d=0.3620, C_{1}=0.1, C_{2}(t):=0$.

Figure 5: $T=3500, \alpha_{s}=0.08, \alpha_{c_{1}}=0.4, \alpha_{c_{2}}=0.4, \gamma_{s}=1, \gamma_{c_{1}}=1, \gamma_{c_{2}}=1, s_{1}=0.3$, $\mu_{2}=800, d=0.3620 ;$ (dashed lines) $C_{1}(t):=0, C_{2}(t):=0 ;$ (dotted lines) $C_{1}(t):=0$, $C_{2}(t):=0.12 ;$ (solid lines) $C_{1}(t):=0.12, C_{2}(t):=0$.

Figure 6: $T=6000, \alpha_{s}=0.08, \alpha_{c_{1}}=0.08, \alpha_{c_{2}}=0.2, \gamma_{s}=1, \gamma_{c_{1}}=1, \gamma_{c_{2}}=1, \mu_{2}=800$, $d=0.3620 ;$ (left) $s_{1}=0.1, C_{1}(t):=0, C_{2}(t):=0 ;$ (center) $s_{1}=0.3, C_{1}(t):=0.07, C_{2}(t):=0$; (right) $s_{1}=0.3, C_{1}(t):=0, C_{2}(t):=0.07$.

Figure 8: $T=6000, \alpha_{s}=0.08, \alpha_{c_{1}}=0.08, \alpha_{c_{2}}=0.2, \gamma_{s}=1, \gamma_{c_{1}}=1, \gamma_{c_{2}}=1, s_{1}=0.3$, $\mu_{2}=800, d=0.3620, C_{1}(t):=0, C_{2}(t):=0$.

Figures 9-10: $T=6000, \alpha_{s}=0.08, \alpha_{c_{1}}=0.08, \alpha_{c_{2}}=0.2, \gamma_{s}=1, \gamma_{c_{1}}=1, \gamma_{c_{2}}=1$, $\mu_{2}=800, d=0.3620, s_{1}=0.3, C_{a}=0.1, C_{b}=2.55$. 
Figures 11: $T=6000, \alpha_{s}=0.08, \alpha_{c_{1}}=0.08, \alpha_{c_{2}}=0.2, \gamma_{s}=1, \gamma_{c_{1}}=1, \gamma_{c_{2}}=1, \mu_{2}=800$, $d=0.3620, s_{1}=0.3, C_{a}=0.05, C_{b}=1.275$.

Figures 12: $T=6000, \alpha_{s}=0.08, \alpha_{c_{1}}=0.08, \alpha_{c_{2}}=0.2, \gamma_{s}=1, \gamma_{c_{1}}=1, \gamma_{c_{2}}=1, \mu_{2}=800$, $d=0.3620, s_{1}=0.3, C_{c}=0.05, C_{d}=1.275$.

\section{References}

[1] A. Arnold, L. Desvillettes, And C. Prévost, Existence of nontrivial steady states for populations structured with respect to space and a continuous trait, Commun. Pure Appl. Anal, 11 (2012), pp. 83-96.

[2] L. Beketic-Oreskovic, G. E. Durán, G. Chen, C. Dumontet, and B. I. Sikic, Decreased mutation rate for cellular resistance to doxorubicin and suppression of MDR1 gene activation by the cyclosporin PSC 833, J. Natl. Cancer Inst., 87 (1995), pp. 15931602 .

[3] I. Beyer, H. Cao, J. Persson, H. Song, M. Richter, Q. Feng, R. Yumul, R. van Rensburg, Z. Li, R. Berenson, ET AL., Coadministration of epithelial junction opener JO-1 improves the efficacy and safety of chemotherapeutic drugs, Clin. Cancer Res., 18 (2012), pp. 3340-3351.

[4] I. Beyer, R. van Rensburg, R. Strauss, Z. Li, H. Wang, J. Persson, R. Yumul, Q. Feng, H. Song, J. BARTeK, Et AL., Epithelial junction opener JO-1 improves monoclonal antibody therapy of cancer, Cancer Res., 71 (2011), pp. 7080-7090.

[5] E. Bouin, V. Calvez, N. Meunier, S. Mirrahimi, B. Perthame, G. Raoul, and R. VoITURIEZ, Invasion fronts with variable motility: phenotype selection, spatial sorting and wave acceleration, C. R. Math., 350 (2012), pp. 761-766.

[6] E. D. BRodie III, Correlational selection for color pattern and antipredator behavior in the garter snake thamnophis ordinoides, Evolution, (1992), pp. 1284-1298.

[7] T. M. Busch, X. Xing, G. Yu, A. Yodh, E. P. Wileyto, H.-W. Wang, T. DurduRAN, T. C. ZHU, AND K. K.-H. WANG, Fluence rate-dependent intratumor heterogeneity in physiologic and cytotoxic responses to Photofrin photodynamic therapy., Photochem. Photobiol. Sci., 8 (2009), pp. 1683-1693.

[8] H. Byrne, Continuum models of avascular tumor growth, in Mathematics and life sciences, A. V. Antoniouk and R. V. N. Melnik, eds., De Gruyter, 2013, ch. 12.1, pp. 279-312.

[9] K. Carver, X. Ming, And R. L. Juliano, Multicellular tumor spheroids as a model for assessing delivery of oligonucleotides in three dimensions, Molecular Therapy-Nucleic Acids, 3 (2014), p. e153.

[10] T. Corbett, D. Griswold, B. Roberts, J. Peckham, and F. Schabel, Tumor induction relationships in development of transplantable cancers of the colon in mice for 
chemotherapy assays, with a note on carcinogen structure, Cancer Res., 35 (1975), pp. 24342439 .

[11] E. C. De Bruin, T. B. Taylor, and C. Swanton, Intra-tumor heterogeneity: lessons from microbial evolution and clinical implications, Genome medicine, 5 (2013), p. 101.

[12] L. Ding, T. J. Ley, D. E. Larson, C. A. Miller, D. C. Koboldt, J. S. Welch, J. K. Ritchey, M. A. Young, T. Lamprecht, M. D. McLellan, et Al., Clonal evolution in relapsed acute myeloid leukaemia revealed by whole-genome sequencing, Nature, 481 (2012), pp. 506-510.

[13] J. Foo, J. Chmielecki, W. Pao, And F. Michor, Effects of pharmacokinetic processes and varied dosing schedules on the dynamics of acquired resistance to erlotinib in EGFRmutant lung cancer, J. Thorac. Oncol., 7 (2012), pp. 1583-1593.

[14] R. Gatenby, A change of strategy in the war on cancer, Nature, 459 (2009), pp. 508-509.

[15] R. Gatenby, A. Silva, R. Gillies, and B. Frieden, Adaptive therapy, Cancer Res., 69 (2009), pp. 4894-4903.

[16] M. Gerlinger, A. J. Rowan, S. Horswell, J. Larkin, D. Endesfelder, E. GronROOS, P. MARTINEZ, AND ET AL., Intratumor heterogeneity and branched evolution revealed by multiregion sequencing, N. Engl. J. Med., 366 (2012), pp. 883-892.

[17] J. Goldie And A. Coldman, Drug resistance in cancer: mechanisms and models, Cambridge University Press, 1998.

[18] M. Gottesman, Mechanisms of cancer drug resistance, Annu. Rev. Med., 53 (2002), pp. 615-627.

[19] A. Grothey, Defining the role of panitumumab in colorectal cancer, Community Oncology, 3 (2006), pp. 10-6.

[20] P. B. Gupta, C. M. Fillmore, G. Jiang, S. D. Shapira, K. Tao, C. Kuperwasser, AND E. S. LANDER, Stochastic state transitions give rise to phenotypic equilibrium in populations of cancer cells., Cell, 146 (2011), pp. 633-644.

[21] M. Inukai, S. Toyooka, S. Ito, H. Asano, S. Ichihara, J. Soh, H. Suehisa, M. Ouchida, K. Aoe, M. Aoe, et AL., Presence of epidermal growth factor receptor gene Tr90M mutation as a minor clone in non-small cell lung cancer, Cancer Res., 66 (2006), pp. 7854-7858.

[22] T. L. Jackson And H. M. Byrne, A mechanical model of tumor encapsulation and transcapsular spread, Math. Biosci., 180 (2002), pp. 307-328.

[23] M. Kimmel And A. Świerniak, Control theory approach to cancer chemotherapy: Benefiting from phase dependence and overcoming drug resistance, in Tutorials in Mathematical Biosciences III, A. Friedman, ed., vol. 1872 of Lecture Notes in Mathematics, Springer Berlin / Heidelberg, 2006, pp. 185-221. 
[24] N. Komarova And D. Wodarz, Drug resistance in cancer: Principles of emergence and prevention, Proc. Natl. Acad. Sci. USA, 102 (2005), pp. 9714-9719.

[25] D. A. Landau, S. L. Carter, P. Stojanov, A. McKenna, K. Stevenson, M. S. Lawrence, C. Sougnez, C. Stewart, A. Sivachenko, L. Wang, et Al., Evolution and impact of subclonal mutations in chronic lymphocytic leukemia, Cell, 152 (2013), pp. $714-726$.

[26] J. Laurent, C. Frongia, M. Cazales, O. Mondesert, B. Ducommun, and V. LobJOIS, Multicellular tumor spheroid models to explore cell cycle checkpoints in 3d, BMC cancer, 13 (2013), pp. 1-12.

[27] O. Lavi, J. M. Greene, D. Levy, and M. M. Gottesman, The role of cell density and intratumoral heterogeneity in multidrug resistance, Cancer Res., 73 (2013), pp. 7168-7175.

[28] U. ŁĘDŻEWICZ AND H. SCHÄTTLER, Optimal bang-bang controls for a two-compartment model in cancer chemotherapy, Jour. Opt. Theory and Appl., 114 (2002), pp. 609-637.

[29] A. Lorz, T. Lorenzi, M. E. Hochberg, J. Clairambault, and B. Perthame, Populational adaptive evolution, chemotherapeutic resistance and multiple anti-cancer therapies, Esaim-Math. Model. Num. Anal., 47 (2013), pp. 377-399.

[30] A. Lorz, S. Mirrahimi, and B. Perthame, Dirac mass dynamics in a multidimensional nonlocal parabolic equation, Commun. Part. Diff. Eq., 36 (2011), pp. 1071-1098.

[31] S. E. Luria And M. DelBrüCK, Mutations of bacteria from virus sensitivity to virus resistance., Genetics, 28 (1943), pp. 491-511.

[32] L. Merlo, J. Pepper, B. Reid, And C. Maley, Cancer as an evolutionary and ecological process, Nat. Rev. Cancer, 6 (2006), pp. 924-935.

[33] A. I. Minchinton and I. F. Tannock, Drug penetration in solid tumours, Nat. Rev. Cancer, 6 (2006), pp. 583-592.

[34] S. Mirrahimi And B. Perthame, Asymptotic analysis of a selection model with space, In preparation, (2014).

[35] S. Mirrahimi and G. Raoul, Dynamics of sexual populations structured by a space variable and a phenotypical trait, Theor. Popul. Biol., 84 (2013), pp. 87-103.

[36] T. Mitchison, The proliferation rate paradox in antimitotic chemotherapy, Mol. Biol. Cell, 23 (2012), pp. 1-6.

[37] K. P. Olive, M. A. Jacobetz, C. J. Davidson, A. Gopinathan, D. Mcintyre, D. Honess, B. Madhu, M. A. Goldgraben, M. E. Caldwell, D. Allard, et al., Inhibition of Hedgehog signaling enhances delivery of chemotherapy in a mouse model of pancreatic cancer, Science, 324 (2009), pp. 1457-1461.

[38] B. Perthame and G. Barles, Dirac concentrations in Lotka-Volterra parabolic PDEs, Indiana Univ. Math. J., 57 (2008), pp. 3275-3301. 
[39] P. B. RAIney AND M. Travisano, Adaptive radiation in a heterogeneous environment, Nature, 394 (1998), pp. 69-72.

[40] K. Scotтo, Transcriptional regulation of abc drug transporters, Oncogene, 22 (2003), pp. 7496-7511.

[41] S. V. Sharma, D. Y. Lee, B. Li, M. P. Quinlan, F. Takahashi, S. Maheswaran, U. McDermott, N. Azizian, L. Zou, M. A. Fischbach, K.-K. Wong, K. Brandstetter, B. Wittner, S. Ramaswamy, M. Classon, and J. Settleman, A chromatin-mediated reversible drug-tolerant state in cancer cell subpopulations., Cell, 141 (2010), pp. 69-80.

[42] A. Silva And R. Gatenby, A theoretical quantitative model for evolution of cancer chemotherapy resistance, Biology Direct, 22 (2010), pp. 5-25.

[43] R. Simon And L. Norton, The Norton-Simon hypothesis: designing more effective and less toxic chemotherapeutic regimens, Nat. Clin. Pract. Oncol., 3 (2006), pp. 406-407.

[44] R. M. Sutherland, Cell and environment interactions in tumor microregions: the multicell spheroid model, Science, 240 (1988), pp. 177-184.

[45] C. Swanton, Intratumor heterogeneity: evolution through space and time, Cancer Res., 72 (2010), pp. $4875-4882$.

[46] G. Szakács, G., J. K. Paterson, J. A. Ludwig, C. Booth-Genthe, and M. M. Gottesman, Targeting multidrug resistance in cancer., Nat. Rev. Drug. Discov., 5 (2006), pp. 219-234.

[47] J. Tabernero, E. Van Cutsem, E. Díaz-Rubio, A. Cervantes, Y. Humblet, T. André, J.-L. Van Laethem, P. Soulié, E. Casado, C. Verslype, J. S. Valera, G. Tortora, F. Ciardiello, O. Kisker, and A. De Gramont, Phase II trial of cetuximab in combination with fluorouracil, leucovorin, and oxaliplatin in the first-line treatment of metastatic colorectal cancer., J. Clin. Oncol., 25 (2007), pp. 5225-5232.

[48] C. Tomasetti And D. Levy, Drug resistance always depends on the turnover rate, in SBEC 2010, IFMBE Proceedings, K. Herold, J. Vossoughi, and W. Bentley, eds., vol. 32, Springer, Berlin, 2010, pp. 552-555.

[49] C. Tomasetti And D. Levy, An elementary approach to modeling drug resistance in cancer, Math. Biosci. Eng., 7 (2010), pp. 905-918.

[50] C.-J. Tsai And R. Nussinov, The molecular basis of targeting protein kinases in cancer therapeutics, in Semin. cancer biol., vol. 23, Elsevier, 2013, pp. 235-242.

[51] P. Vaupel And M. Hockel, Blood supply, oxygenation status and metabolic micromilieu of breast cancers: characterization and therapeutic relevance., Int. J. Oncol., 17 (2000), pp. 869-948.

[52] P. Vaupel, F. Kallinowski, And P. OkuniefF, Blood flow, oxygen and nutrient supply, and metabolic microenvironment of human tumors: a review, Cancer Res., 49 (1989), pp. 6449-6465. 
[53] R. Weinberg, The Biology of Cancer, Garland Science, 2007.

[54] I. B. Weinstein And A. Joe, Oncogene addiction, Cancer Res., 68 (2008), pp. 3077-3080.

[55] L.-C. Ye, T.-S. Liu, L. Ren, Y. Wei, D.-X. Zhu, S.-Y. Zai, Q.-H. Ye, Y. Yu, B. XU, X.-Y. QIN, AND J. XU, Randomized controlled trial of cetuximab plus chemotherapy for patients with KRAS wild-type unresectable colorectal liver-limited metastases., J. Clin. Oncol., 31 (2013), pp. 1931-1938.

[56] P. Yu, M. Mustata, L. Peng, J. J. Turek, M. R. Melloch, P. M. French, AND D. D. NolTe, Holographic optical coherence imaging of rat osteogenic sarcoma tumor spheroids, Appl. Opt., 43 (2004), pp. 4862-4873.

[57] D. C. Zhou, S. Ramond, F. Viguié, A. M. Faussat, R. Zittoun, and J. P. Marie, Sequential emergence of MRP- and MDR1-gene over-expression as well as MDR1-gene translocation in homoharringtonine-selected K562 human leukemia cell lines., Int. J. Cancer, 65 (1996), pp. 365-371. 
$\int \mathrm{n}(\mathrm{t}, \mathrm{r}, \mathrm{x}) \mathrm{dr} / \rho_{\mathrm{T}}(\mathrm{t})$ at $\mathrm{t}=\mathrm{T}$

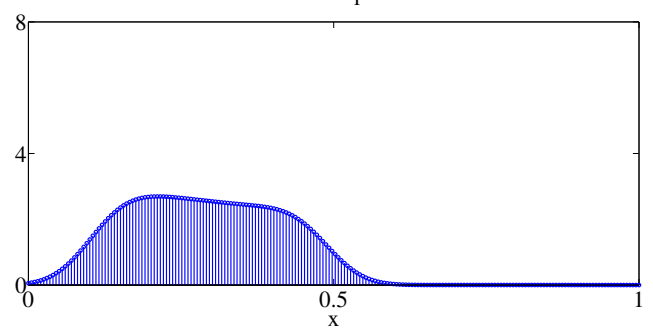

$\int \mathrm{n}(\mathrm{t}, \mathrm{r}, \mathrm{x}) \mathrm{dr} / \rho_{\mathrm{T}}(\mathrm{t})$ at $\mathrm{t}=\mathrm{T}$

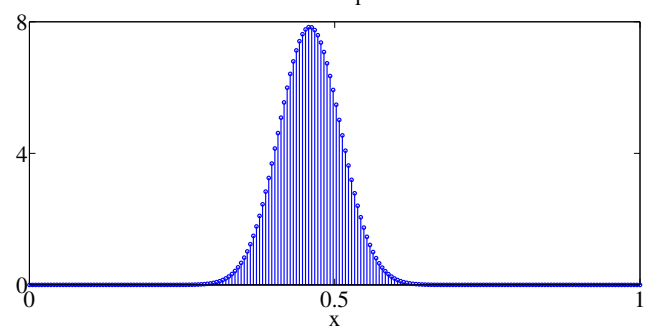

(b)

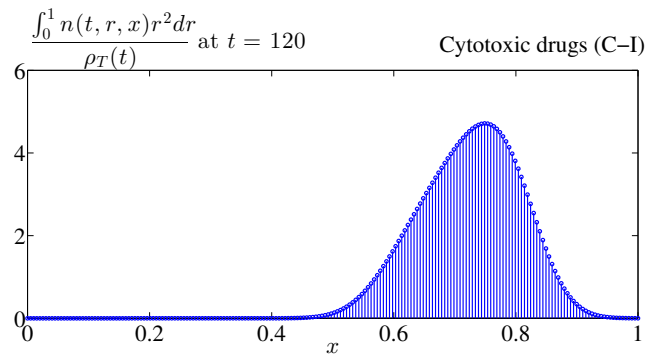

(a)
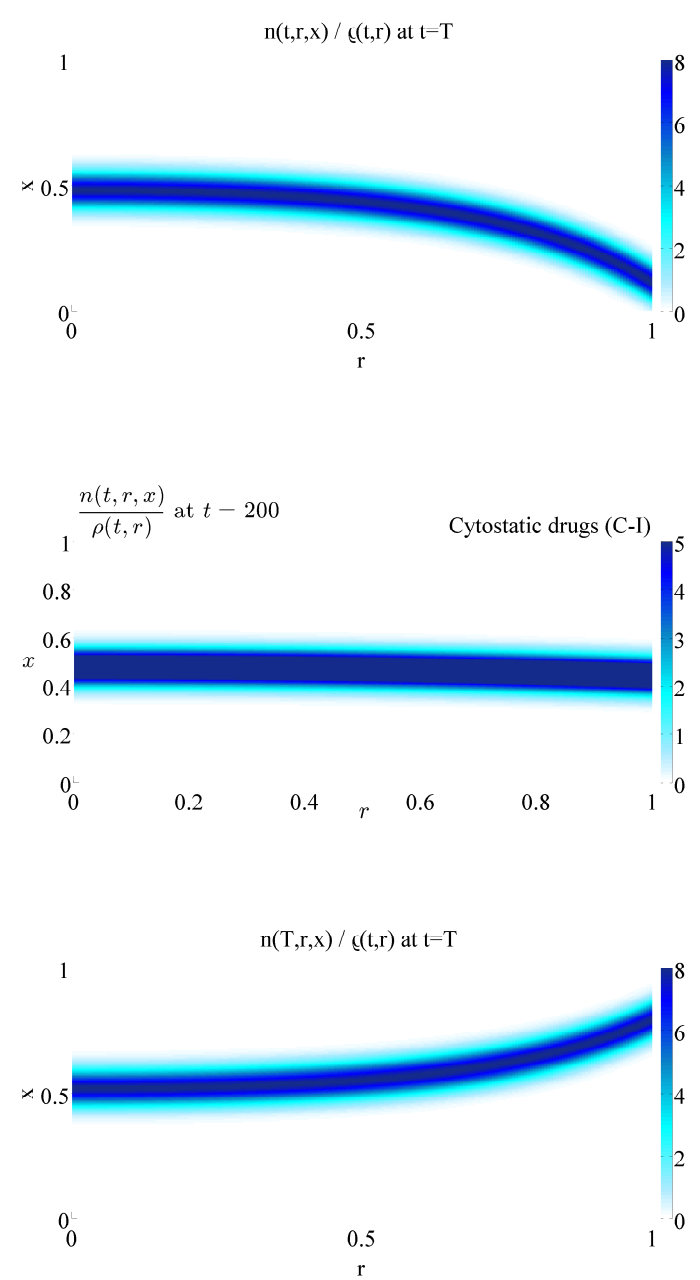

(c)

Figure 2: Cell environmental adaptation.

(a) Without drugs. Plots of $\int_{0}^{1} n(t, r, x) r^{2} d r / \varrho_{T}(t)$ (left panel) and $n(t, r, x) / \varrho(t, r)$ (right panel) at $t=50$ for $C_{1,2}(t):=0$. For each $r$ value, the function $n(t, r, x)$ concentrates in a different point $X(t, r)$. Cells characterized by a low expression level of resistance to cytotoxic therapies and by a strong proliferative potential are selected, and this is particularly obvious at the rim of the spheroid $(r=1)$, where nutrients abound. (b) Under constant infusion of cytostatic drugs. Plots of $\int_{0}^{1} n(t, r, x) r^{2} d r / \varrho_{T}(t)$ (left panel) and $n(t, r, x) / \varrho(t, r)$ (right panel) at $t=200$ for $C_{1}(t):=0$ and $C_{2}(t):=C_{2}>0$. The cell distribution at $t=200$ is still close to the initial one (see Fig. 1), that is, cytostatic drugs slow down the evolution of cancer cells. The unit of time is days. (c) Under constant infusion of cytotoxic drugs. Plots of $\int_{0}^{1} n(t, r, x) r^{2} d r / \varrho_{T}(t)$ (left panel) and $n(t, r, x) / \varrho(t, r)$ (right panel) at $t=120$ for $C_{1}(t):=C_{1}>0$ and $C_{2}(t):=0$. For each value of $r$, the function $n(t, r, x)$ concentrates in a different point $X(t, r)$. Cells characterized by high resistance levels are selected. As in the case without drugs, this is particularly obvious at the rim of the spheroid $(r=1)$, where drugs abound. Details of the parameters used are provided in Appendix B. 

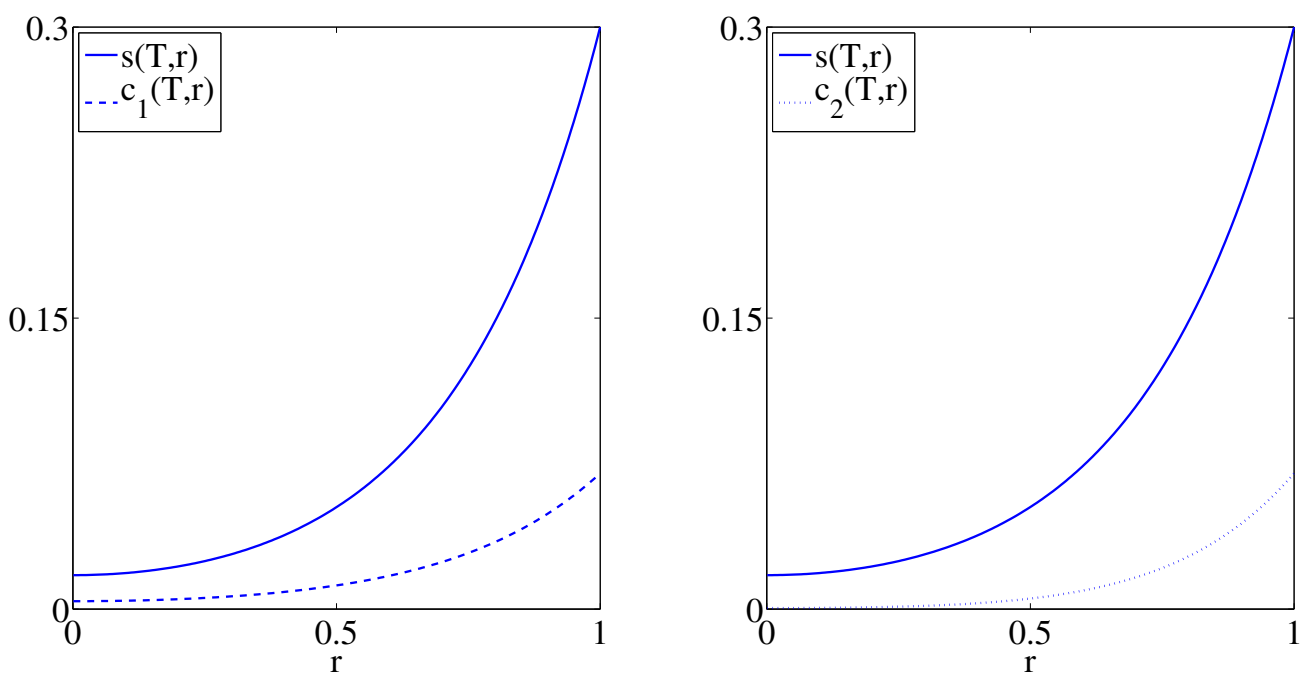

Figure 3: Distributions of resources and drugs. Plots of $s(t, r)$ (solid line) and $c_{1}(t, r)$ (dashed line) at $t=120$, for $C_{1}(\cdot):=C_{1}>0, C_{2}(\cdot)=0$ (left panel). Plots of $s(t, r)$ (solid line) and $c_{2}(t, r)$ (dotted line) at $t=200$, for $C_{1}(\cdot):=0, C_{2}(\cdot):=C_{2}>0$ (left panel). The plot of $s(t, r)$ at $t=50$ in the case $C_{1.2}(\cdot):=0$ is analogous to that presented here. The unit of time is days. Details of the parameters used are provided in Appendix B.

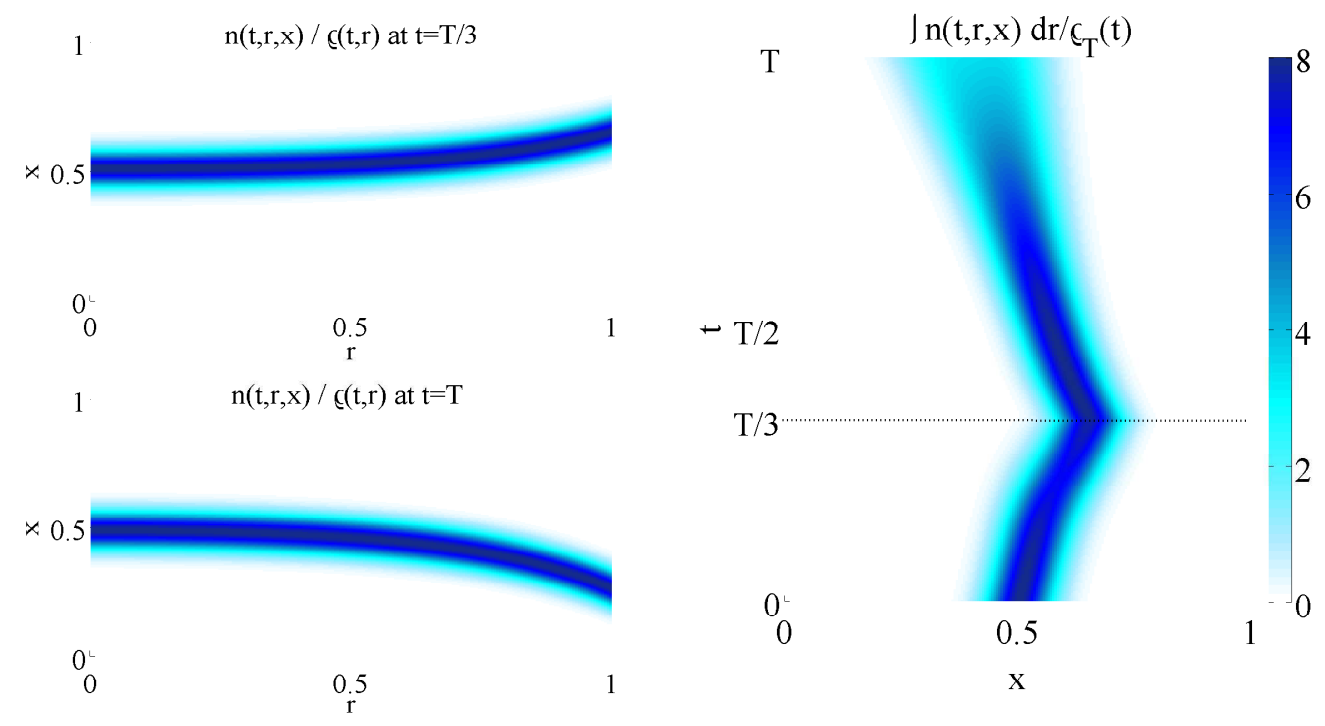

Figure 4: On-off switch of cytotoxic drug infusion. Plots of $n(t, r, x) / \varrho(t, r)$ at $t=20$ (left-top panel), $n(t, r, x) / \varrho(t, r)$ at $t=60$ (left-bottom panel) and $\int_{0}^{1} n(t, r, x) r^{2} d r / \varrho_{T}(t)$ with $t \in[0,60]$ (right panel), for $C_{1}(t)$ defined by $(4.1)$ and $C_{2}(t):=0$. The selection of higher levels of resistance occurs during the infusion of cytotoxic drugs, i.e., in the time interval [0,20], while higher levels of proliferative potential are selected in the absence of cytotoxic drugs, i.e., in the time interval $(20,60]$. The unit of time is days. Details of the parameters used are provided in Appendix B. 


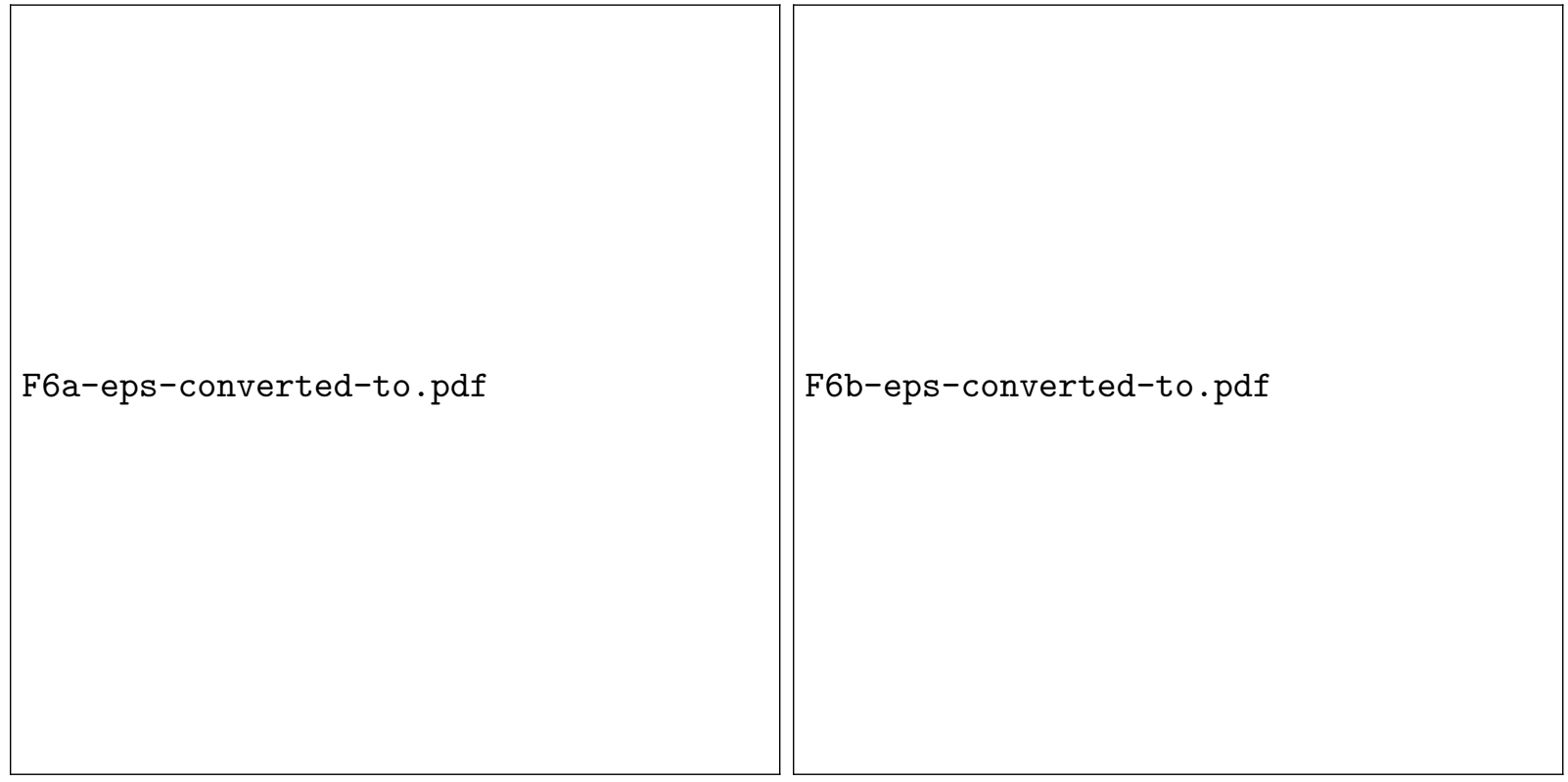

(a)

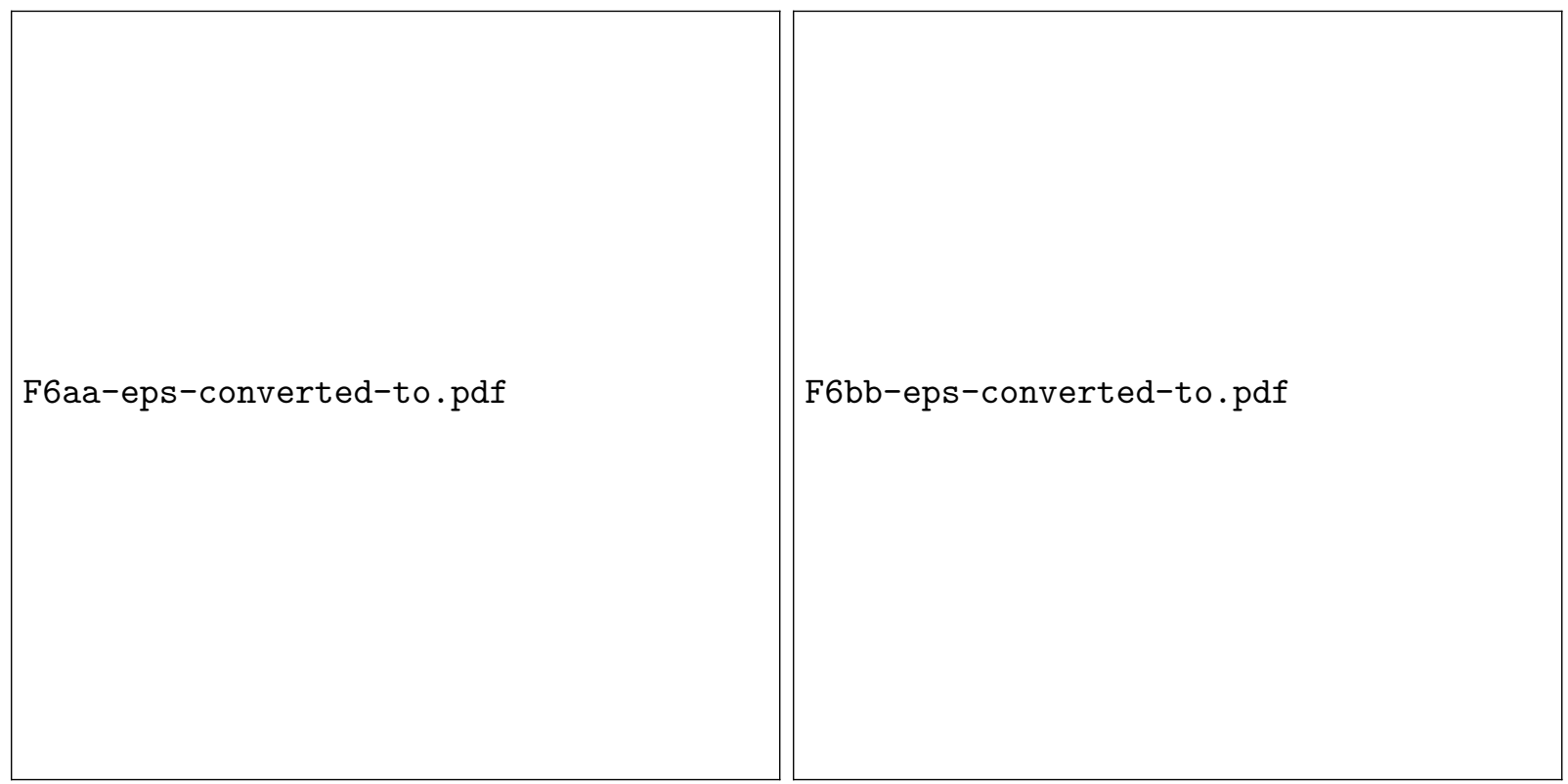

(b)

Figure 5: Evolution of the global average level of resistance and related variance. (a) Under constant infusion of cytostatic drugs. Plot of $\chi(t)$ (left panel) and $\sigma^{2}(t)$ (right panel) for $C_{1,2}(t):=0$ (solid lines), $C_{1}(t):=C_{1}>0, C_{2}(t):=0$ (dashed lines) and $C_{1}(t):=0, C_{2}(t):=C_{2}>0$ (dotted lines). (c) Under constant infusion of cytostatic drugs. Cytotoxic drugs increase the average level of resistance $\chi(t)$ over time, while the related variance $\sigma^{2}(t)$ decreases. This may be interpreted as a reduction of intra-tumor heterogeneity w.r.t. the resistance trait, due to the second selection pressure introduced by the cytotoxic drugs. The unit of time is days. Details of the parameters used are provided in Appendix B. 

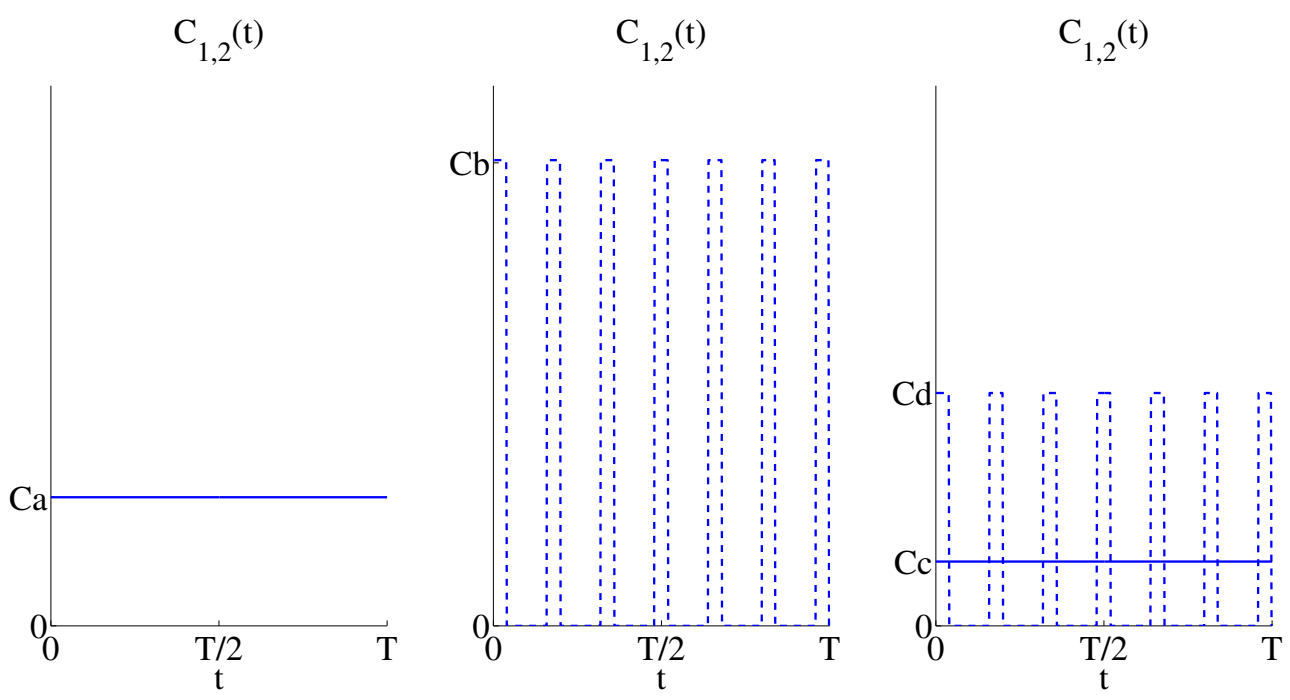

Figure 6: Infusion schemes of cytotoxic and cytostatic drugs. Definitions of boundary conditions $C_{1,2}(t)$. The left and center panels refer to constant and bang-bang infusion of cytotoxic and/or cytostatic drugs, while the right panel refers to the case where cytotoxic drugs are delivered through a bang-bang infusion scheme while cytostatic drugs are constantly supplied, or vice-versa. The parameters $C_{a, b, c, d}$ model the instantaneous delivered doses. The unit of time is days. 


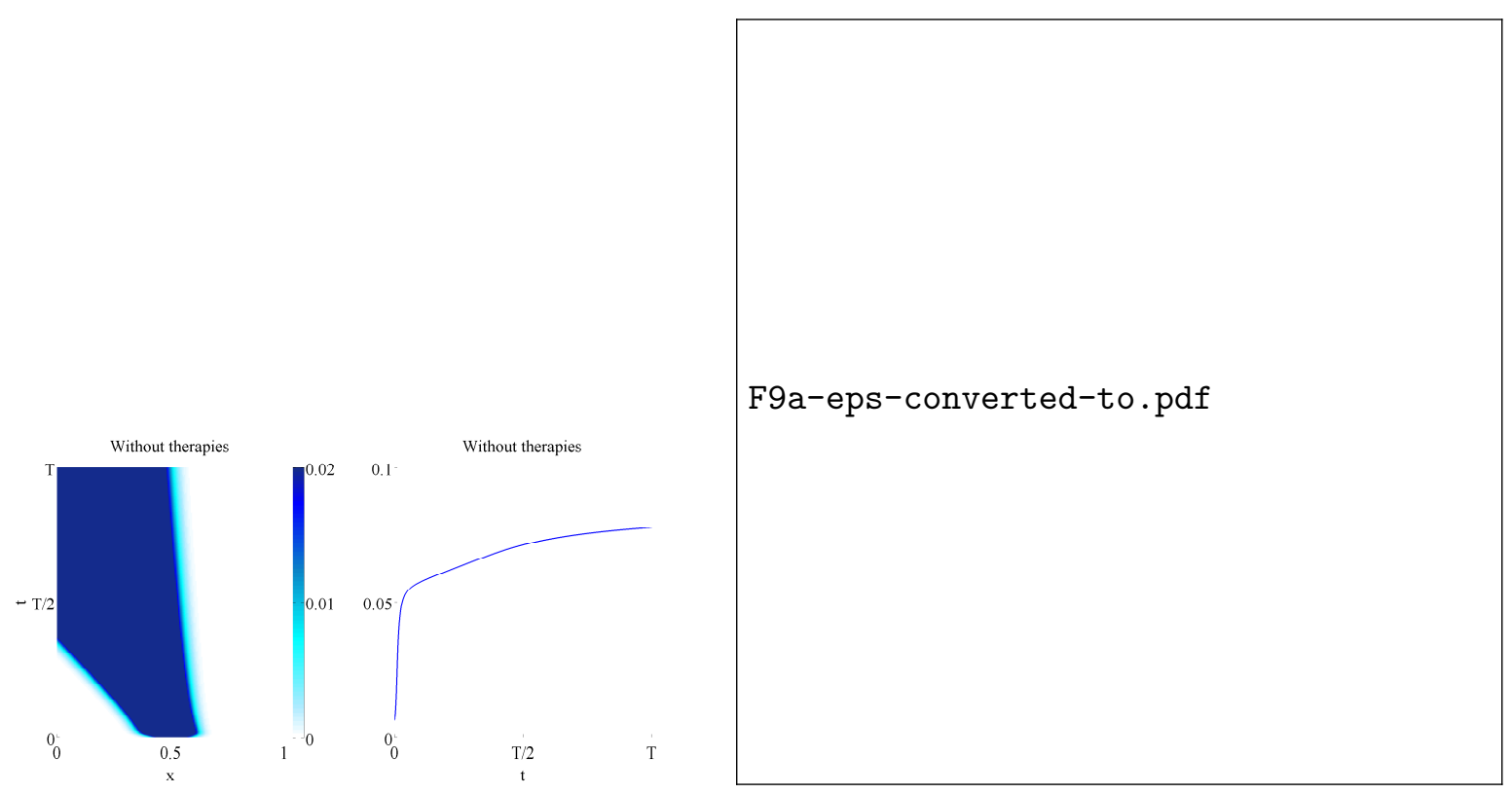

(a)

Figure 7: Cell dynamics without drugs. Plots of $\int_{0}^{1} n(t, r, x) r^{2} d r$ (left panel) and $\varrho_{T}(t)$ (right panel). In agreement with the results presented in Section 3, cells characterized by a low expression level of resistance to cytotoxic therapies (i.e., strong proliferative potentials) are selected and intra-tumor heterogeneity is high. The unit of time is days. All values are normalized with respect to the initial global population density. To be compared with Figs. 811 . 

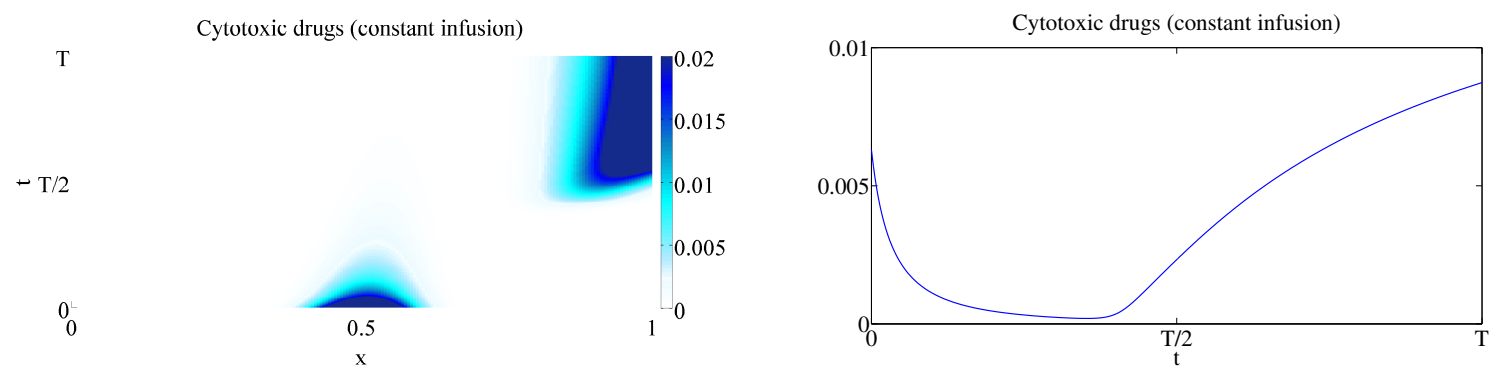

(a)
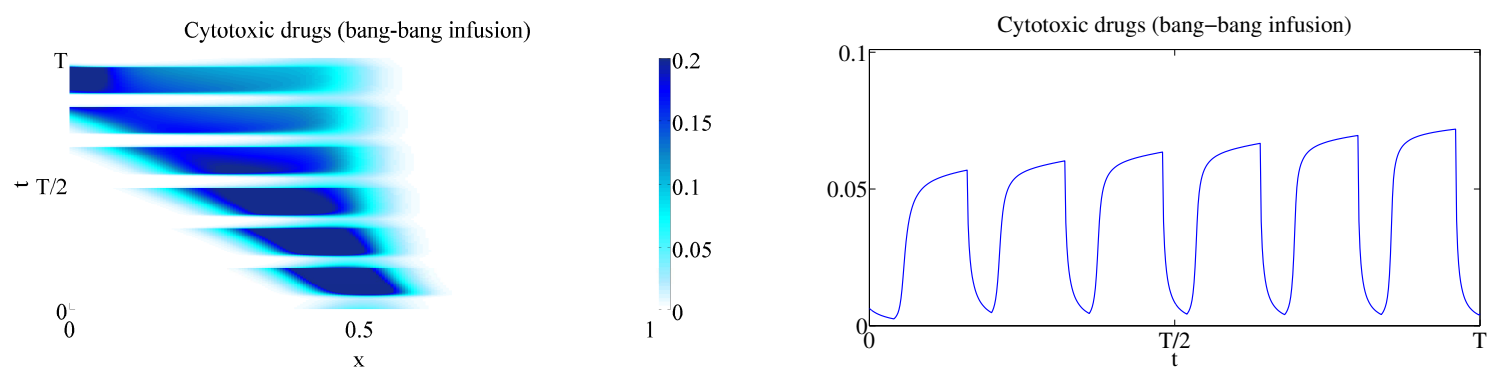

(b)

Figure 8: (a) Constant infusion of cytotoxic drugs only. Plots of $\int_{0}^{1} n(t, r, x) r^{2} d r$ (left panel) and $\varrho_{T}(t)$ (right panel). The constant infusion of cytotoxic drugs leads to a temporary reduction of the population density of cancer cells, which is followed by tumor relapse. (b) Bang-bang infusion of cytotoxic drugs only. Plots of $\int_{0}^{1} n(t, r, x) r^{2} d r$ (left panel) and $\varrho_{T}(t)$ (right panel). The bang-bang infusion of cytotoxic drugs weakly affects the dynamics of cancer cells by comparison with the case without therapies, apart from a temporary reduction of the population density of cancer cells during drug delivery. The unit of time is days. All values are normalized with respect to the initial global population density. Details of the parameters used are provided in Appendix B. 

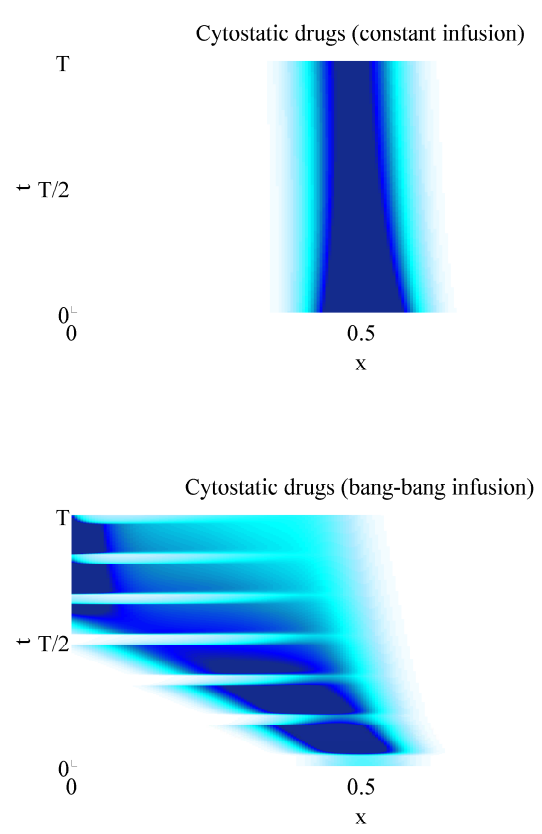
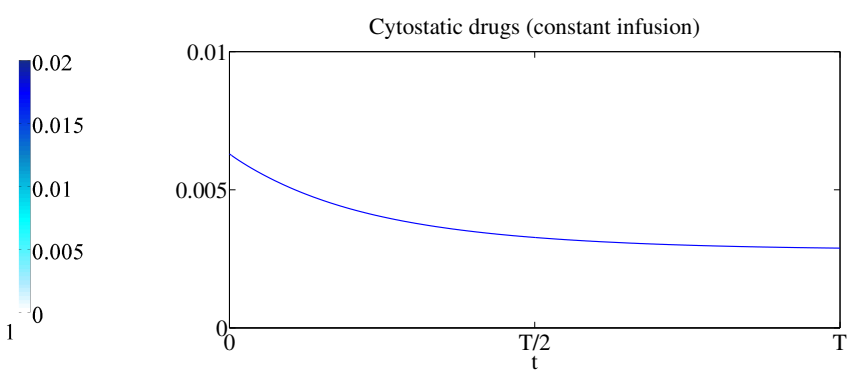

(a)
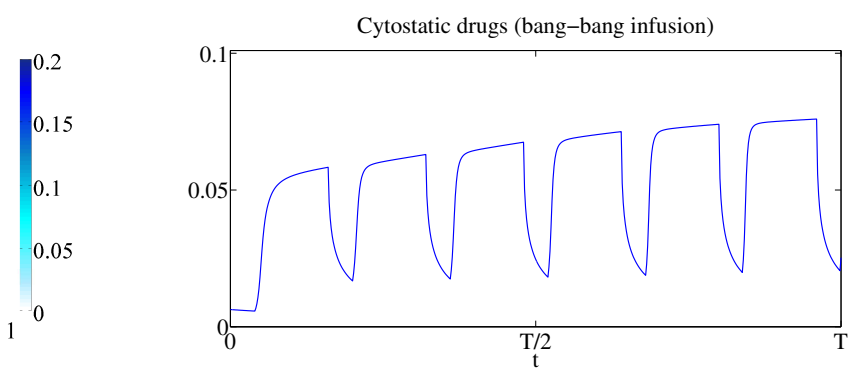

(b)

Figure 9: (a) Constant infusion of cytostatic drugs only. Plots of $\int_{0}^{1} n(t, r, x) r^{2} d r$ (left panel) and $\varrho_{T}(t)$ (right panel). The constant infusion of cytostatic drugs tends to slow down the selection of high proliferative potentials by comparison with the case without drugs. (b) Bang-bang infusion of cytostatic drugs only. Plots of $\int_{0}^{1} n(t, r, x) r^{2} d r$ (left panel) and $\varrho_{T}(t)$ (right panel). The bang-bang infusion of cytotoxic drugs weakly affects the dynamics of cancer cells by comparison with the case without therapies, apart from a temporary reduction of the population density of cancer cells during drug delivery. The unit of time is days. All values are normalized with respect to the initial global population density. Details of the parameters used are provided in Appendix B. 

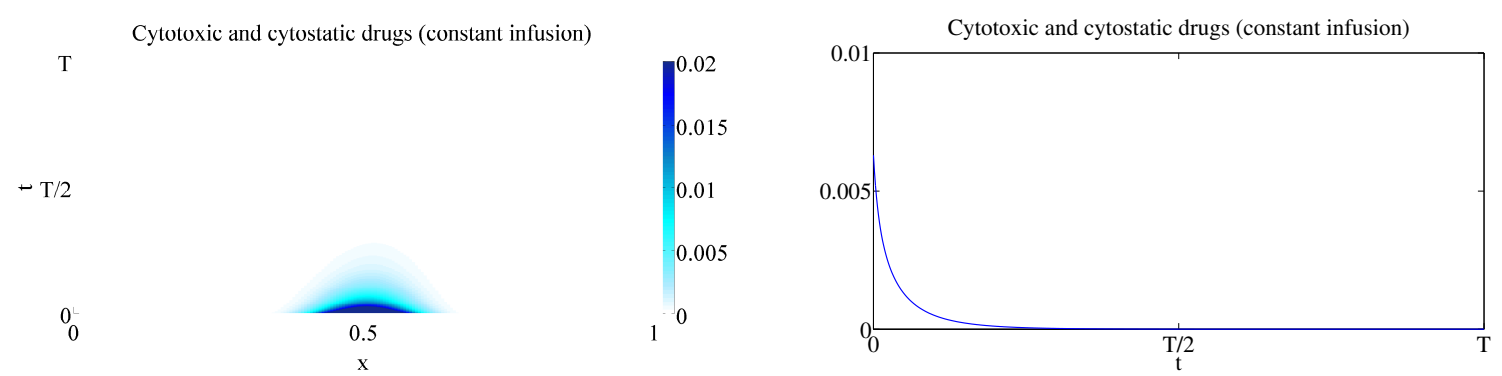

(a)
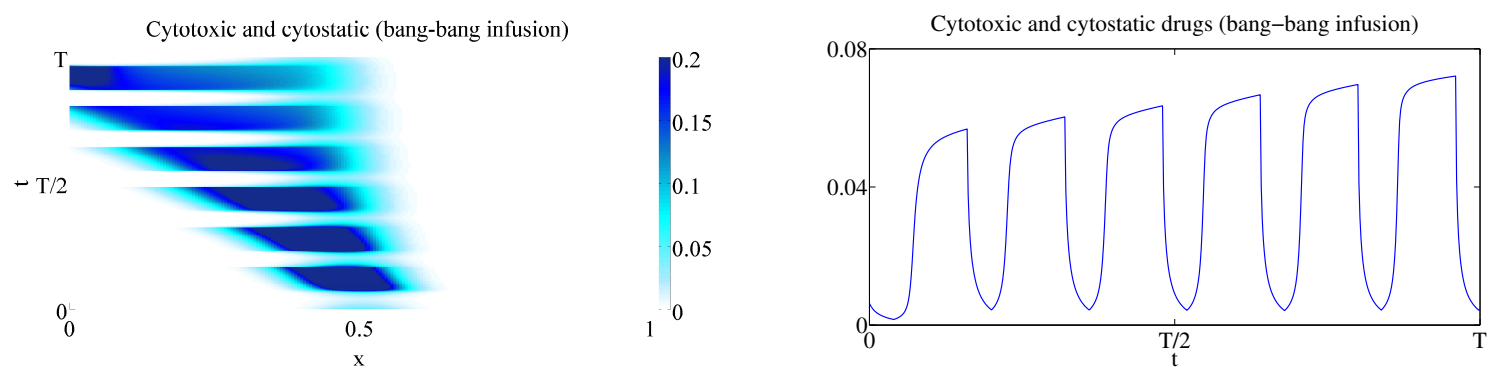

(b)

Figure 10: (a) Constant infusion of cytotoxic and cytostatic drugs. Plots of $\int_{0}^{1} n(t, r, x) r^{2} d r$ (left panel) and $\varrho_{T}(t)$ (right panel). The constant infusion of cytotoxic and cytostatic drugs pushes cancer cells toward extinction. (b) Bang-bang infusion of cytotoxic and cytostatic drugs. Plots of $\int_{0}^{1} n(t, r, x) r^{2} d r$ (left panel) and $\varrho_{T}(t)$ (right panel). The bangbang infusion of cytotoxic and cytostatic drugs weakly affects the dynamics of cancer cells by comparison with the case without therapies, apart from temporary reductions of the population density of cancer cells during drug delivery. The unit of time is days. All values are normalized with respect to the initial global population density. Details of the parameters used are provided in Appendix B. 

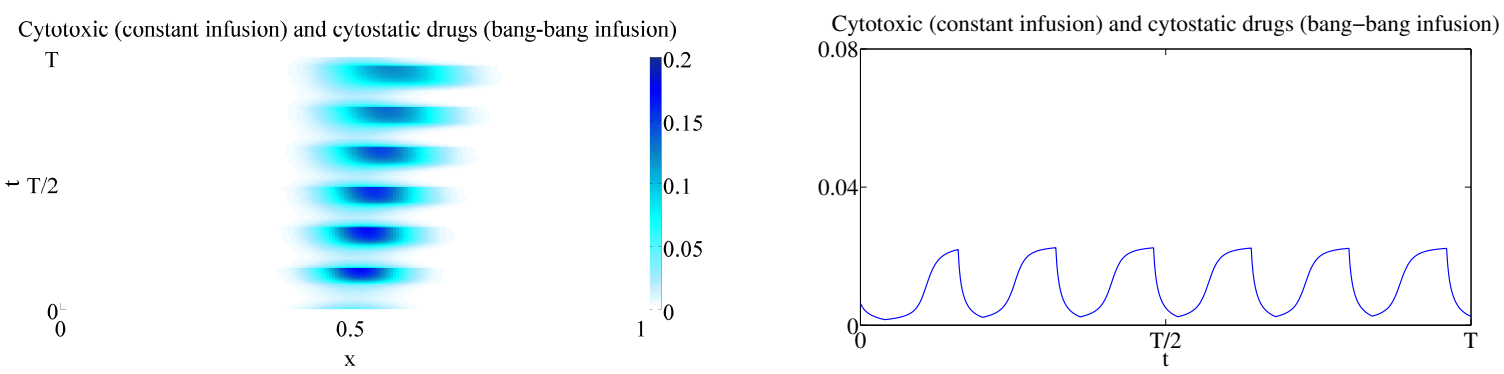

(a)
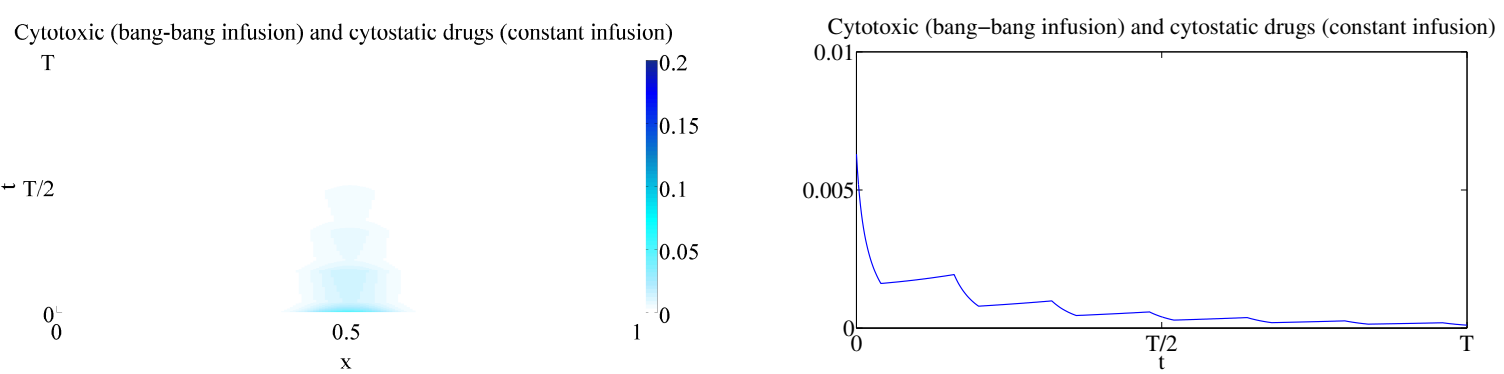

(b)

Figure 11: (a) Constant infusion of cytotoxic drugs and bang-bang infusion of cytostatic drugs. Plots of $\int_{0}^{1} n(t, r, x) r^{2} d r$ (left panel) and $\varrho_{T}(t)$ (right panel). Bang-bang infusion of cytostatic drugs together with constant infusion of cytotoxic drugs makes possible a control on tumor size, but a detectable number of cancer cells survives within the population. (b) Bang-bang infusion of cytotoxic drugs and constant infusion of cytostatic drugs. Plots of $\int_{0}^{1} n(t, r, x) r^{2} d r$ (left panel) and $\varrho_{T}(t)$ (right panel). Bang-bang infusion of cytotoxic drugs together with constant delivery of cytostatic drugs can push cancer cells toward extinction. The unit of time is days. All values are normalized with respect to the initial global population density. Details of the parameters used are provided in Appendix B. 

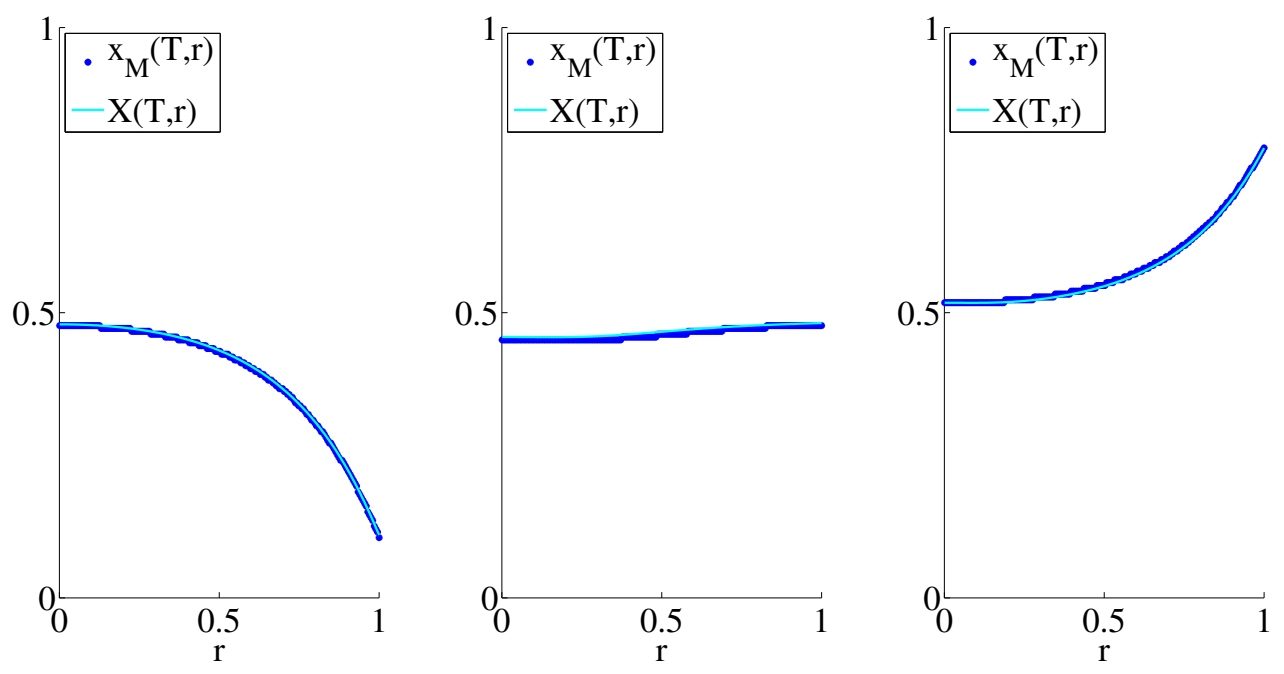

Figure 12: Qualitative mathematical justification for phenotypic selection. Plots of $X(T, r)$ (solid lines) and positions of the maximum points $x_{M}(T, r)$ of $n(T, r, x) / \varrho(T, r)(\bullet)$ for $C_{1,2}(t):=0$ (left panel), $C_{1}(t):=C_{1}>0, C_{2}(t):=0$ (center panel), and $C_{1}(t):=0$, $C_{2}(t):=C_{2}>0$ (right panel). 